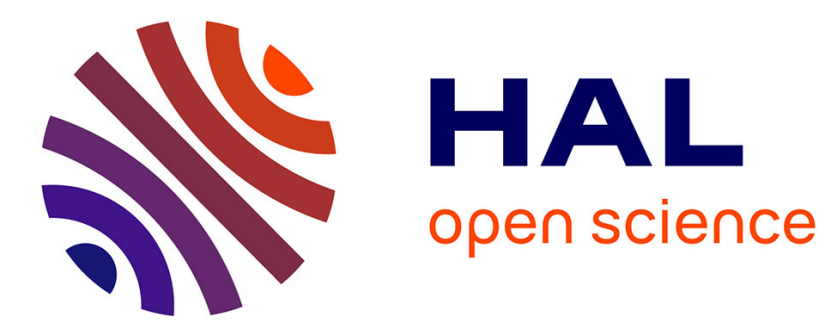

\title{
A Cross-layer Framework for Multiobjective Performance Evaluation of Wireless Ad Hoc Networks
}

Katia Jaffrès-Runser, Mary Schurgot, Qi Wang, Cristina Comaniciu, Jean-Marie Gorce

\section{- To cite this version:}

Katia Jaffrès-Runser, Mary Schurgot, Qi Wang, Cristina Comaniciu, Jean-Marie Gorce. A Crosslayer Framework for Multiobjective Performance Evaluation of Wireless Ad Hoc Networks. Ad Hoc Networks, 2013, vol. 11 (nº 8), pp. 2147-2171. 10.1016/j.adhoc.2013.04.006 . hal-01130011

\section{HAL Id: hal-01130011 \\ https://hal.science/hal-01130011}

Submitted on 11 Mar 2015

HAL is a multi-disciplinary open access archive for the deposit and dissemination of scientific research documents, whether they are published or not. The documents may come from teaching and research institutions in France or abroad, or from public or private research centers.
L'archive ouverte pluridisciplinaire HAL, est destinée au dépôt et à la diffusion de documents scientifiques de niveau recherche, publiés ou non, émanant des établissements d'enseignement et de recherche français ou étrangers, des laboratoires publics ou privés. 


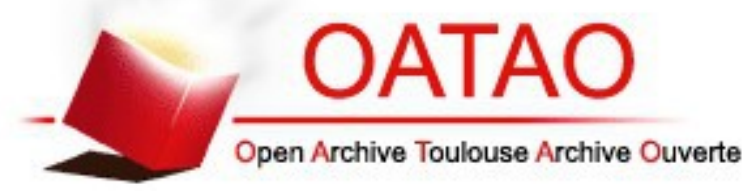

\section{Open Archive TOULOUSE Archive Ouverte (OATAO)}

OATAO is an open access repository that collects the work of Toulouse researchers and makes it freely available over the web where possible.

This is an author-deposited version published in : http://oatao.univ-toulouse.fr/ Eprints ID : 12501

To link to this article : DOI :10.1016/j.adhoc.2013.04.006 URL : http://dx.doi.org/10.1016/j.adhoc.2013.04.006

To cite this version : Jaffres-Runser, Katia and Schurgot, Mary and Wang, Qi and Comaniciu, Cristina and Gorce, Jean-Marie $\underline{A \text { Cross- }}$ layer Framework for Multiobjective Performance Evaluation of Wireless Ad Hoc Networks. (2013) Ad Hoc Networks, vol. 11 ( $\left.\mathrm{n}^{\circ} 8\right)$. pp. 2147-2171. ISSN 1570-8705

Any correspondance concerning this service should be sent to the repository administrator: staff-oatao@,listes-diff.inp-toulouse.fr 


\title{
A cross-layer framework for multiobjective performance evaluation of wireless ad hoc networks
}

\author{
Katia Jaffrès-Runser ${ }^{\mathrm{a}, \mathrm{b}, *}$, Mary R. Schurgot ${ }^{\mathrm{a}, 1}$, Qi Wang ${ }^{\mathrm{d}, \mathrm{c}}$, Cristina Comaniciu ${ }^{\mathrm{a}}$, \\ Jean-Marie Gorce ${ }^{c}$ \\ ${ }^{a}$ Dept. of Electrical and Computer Engineering, Stevens Institute of Technology, Hoboken, NJ 07030, USA \\ ${ }^{\mathrm{b}}$ Université de Toulouse, INPT-ENSEEIHT/IRIT, Toulouse, France \\ ${ }^{\mathrm{c}}$ Université de Lyon, INRIA/INSA-Lyon, CITI, Lyon, France \\ ${ }^{\mathrm{d}}$ Institute of Computing Technology, Chinese Academy of Sciences, Beijing, China
}

\begin{abstract}
A B S T R A C T
In this paper we address the problem of finding the optimal performance region of a wireless ad hoc network when multiple performance metrics are considered. Our contribution is to propose a novel cross-layer framework for deriving the Pareto optimal performance bounds for the network. These Pareto bounds provide key information for understanding the network behavior and the performance trade-offs when multiple criteria are relevant. Our approach is to take a holistic view of the network that captures the cross-interactions among interference management techniques implemented at various layers of the protocol stack (e.g. routing and resource allocation) and determines the objective functions for the multiple criteria to be optimized. The resulting complex multiobjective optimization problem is then solved by multiobjective search techniques. The Pareto optimal sets for an example sensor network are presented and analyzed when delay, reliability and energy objectives are considered.
\end{abstract}

\section{Introduction}

Wireless ad hoc and sensor networks often operate in difficult environments and require several performance criteria to be satisfied related to the timely, reliable, and secure exchange of data. To enable communication across the network, key design elements include routing and resource allocation protocols. Various constraints related to transmission delay [1], energy consumption [2] or fairness

\footnotetext{
* Corresponding author. Address: Université de Toulouse, INPT-ENS EEIHT/IRIT, 2 rue Charles Camichel, BP 7122, 31061 Toulouse Cedex 7 France. Tel.: +33534322190.

E-mail addresses: katia.jaffres-runser@irit.fr (K. Jaffrès-Runser), maschurg@lgsinnovations.com (M.R. Schurgot), wangqi08@ict.ac.cn (Q. Wang), ccomanic@stevens.edu (C. Comaniciu), jean-marie.gorce@insalyon.fr (J.-M. Gorce).

${ }^{1}$ Mary R. Schurgot is now with LGS Bell Labs Innovations. This work was completed while a Ph.D. candidate in the Dept. of Electrical and Computer Engineering at Stevens Institute of Technology.
}

$[3,4]$ are added to the protocol's main design goal of reliable information transfer. Thus, the assessment of such protocol performance relies on various criteria measures which may be evaluated analytically or through network simulations.

It is possible to understand the capabilities and limits of a routing protocol or a resource allocation strategy if a bound on the network performance is known. An end user requires certain simultaneous levels of performance for multiple criteria to guarantee quality of service, yet the considered performance metrics often conflict. The solution maximizing for instance capacity may not be the one that minimizes delay. Therefore all of these performance metrics have to be considered concurrently in the optimization process. In the case of multiple criteria, however, there is usually no single optimum and several solutions offering different optimal trade-offs exist. More specifically, if you decide to optimize delay and capacity, you will 
find solutions (i.e. routing and resource allocation strategies) in the Pareto optimal set that favor either capacity, delay, or some trade-off combination. The main contribution of this paper is to propose a unified cross-layer framework capable of capturing the trade-offs existing between multiple performance metrics.

The solution we propose for obtaining bounds on multiple benchmark objectives relies on the definition of a multiobjective optimization problem; the subsequent evaluation provides a set of Pareto optimal solutions. The Pareto set is composed of non-dominated solutions, i.e. solutions of the search space that are never dominated by any other with respect to the evaluation criteria considered. A solution herein characterizes one possible route configuration with its resource allocation defined in terms of transmission rate. For example, a possible solution to facilitate the transfer of information from source to destination could be the selection of an intermediate node to relay the data with a fixed transmission rate. This multiobjective optimization problem is hard to solve since it is the combination of a resource allocation problem and a routing problem. Compared to analytical approaches, it has the drawback of being computationally more expensive but has two main advantages. First, it can be used to derive bounds for multiple performance metrics at a time. Second, it not only provides the performance bounds, but also extracts the solutions that are Pareto optimal which cannot be determined using a classic analytical approach.

In order to mitigate the limitations of the layered network architecture, cross-layer interactions have to be considered in the framework definition. In [5], the authors address a cross-layer optimization problem of joint design of routing, Medium Access Control (MAC), and physical layer protocols with cooperative communication to achieve minimum power cost under a specified per-hop packet error rate (PER) objective in wireless sensor networks. The authors in [6] highlight the need to find "a simple interface to the physical layer that allows the upper layers to achieve optimal or near optimal cross-layer performance based on the underlying channel conditions". For the cross-layer model we describe in the following sections, we define a link probability-the probability a packet arrives over a given link. This parameter serves as an interface between network layer routing/link layer management decisions and expected physical layer performance by leveraging the broadcast nature of wireless transmissions. The variables of our multi-objective optimization problem determine how often and when a node should re-broadcast a received packet. The approach we take in this work provides for the characterization of fundamental upper bounds, the tradeoff space of multiple criteria, and the routing and resource allocation decisions to achieve these tradeoffs. This approach mirrors the key research areas proposed in the framework of [6]. The authors describe the need for joint research in the areas of fundamental performance upper bounds, layerless dynamic network performance, and application and network optimization. It is through the interaction of these research areas that ad hoc network design and performance can be related and formalized.

Understanding the trade-offs involved with various routing solutions will enable adaptive resource manage- ment across layers and nodes, leading to a more accurate "local to global performance mapping" for practical routing protocol design. The identification of Pareto optimal solutions provides not only achievable performance bounds, but also specific solution sets for the routing and resource allocation algorithms to operate at those bounds. In this paper, we propose a novel framework capable of providing a bound for joint optimization of multiple performance metrics. Our proposed framework comprises both a probabilistic cross-layer network model and a multiobjective optimization problem formulation. Our proposed cross-layer network model captures more accurately the interactions between routing decisions and resource allocation, assuming a basic random medium access control protocol. With its intrinsic probabilistic definition, it is capable of defining various routing techniques such as multi-hop single path routes, broadcast protocols or multi-path protocols. The multiobjective optimization problem is solved using the Parallel Multiobjective Tabu Search (PMOTS) algorithm to retrieve the set of Pareto optimal solutions. This global cross-layer multiobjective framework is applied herein to tackle the problem of robust routing and resource allocation for wireless sensor networks. The following three criteria are relevant in this context: (i) reliability defined as the probability of having a successful packet transmission, (ii) delay, defined as the average end-to-end delay in the network, and (iii) the forwarding energy, defined as the energy spent by the network for relaying.

The goal of our work is different from other approaches aimed to distributively compute Pareto optimal routing algorithms with respect to multiple performance criteria as proposed in $[7,8]$. Our aim is not to develop a new routing algorithm, but to provide a general framework capable of capturing the performance trade-offs of a given network by computing the set of Pareto optimal routing strategies. This characterization provides an efficient tool to:

- compare the performance of existing routing algorithms to the bound provided by the set of Pareto optimal strategies, and

- foster the development of more efficient and flexible routing strategies based on end-user requirements for network performance.

Our main contributions in this work are twofold:

1. Propose a general cross-layer framework network model capable of capturing the impact and interaction of a wide range of interference and resource management techniques for various channel conditions.

2. Formulate a multiobjective routing optimization problem by defining appropriate evaluation functions for criteria such as reliability of information transfer, endto-end delay, and energy consumption.

The multiobjective routing optimization problem described in the following can be solved using existing multiobjective search techniques [9]. Our paper focuses mostly on the derivation of the proposed cross-layer framework, and gives only a brief description of the optimization 
heuristic considered for solving the problem. A first description of our model can be found in [10]. The formulation given in the present paper provides a more detailed description of our complete framework and extends the results of [10] in several ways, providing an in-depth analysis of the framework and the multiobjective bounds derived with it. This articles aims at understanding the nature of the bounds calculated by analyzing comprehensively the Pareto optimal solutions sets composing it. It extends as well the work of [10] by assessing the analytical results with simulations, studying the impact of the network geometry on the bounds and using the bounds to compare the performance of well-known routing strategies such as AODV and DSR.

The paper is organized as follows. Related work is presented in Section 2. In Section 3 we present our cross-layer framework based on a probabilistic network model. Section 4 formulates routing and resource allocation in an ad hoc network as a multiobjective optimization problem and Section 5 discusses an example for sensor networks. Results and analysis for two problem instances are then given in Section 6 to illustrate our modeling framework. Obtained bounds are assessed using simulations and compared to solutions provided by standard ad hoc routing protocols. Section 7 concludes the paper.

\section{Related work}

Over the last decades, research efforts have studied wireless ad hoc network performance from at least three perspectives:

1. deriving analytical upper bounds and scaling laws on performance,

2. constructing and solving single objective functions for network optimization, and

3. formulating and solving multiobjective optimization problems to capture performance tradeoffs.

The first point emphasizes on defining closed form expressions of a network performance metric, usually as a function of the size of the network. Such bounds are essential to the understanding of the fundamental limits of wireless networking. The two other points take a closer look at the problem by formulating an optimization problem (mono- or multiobjective) capable of extracting a very tight bound on the considered performance metrics. Even though analytical scaling laws are usually very fast to compute, they provide looser upper bounds than optimizationbased formulations do. Indeed, these more computationally expensive approaches provide a really tight (almost exact) upper bound and have the second advantage of deriving at the same time optimal network configurations. Centralized and distributed optimization solutions have been considered, the latter ones being mostly studied for their straightforward practicality. However, distributed solutions may not completely converge to optimality. Thus, a truthful performance bound is obtained through centralized optimization which is the approach considered in this paper. The first part of this related work section highlights main works on analytical models while the second one presents studies related to centralized optimization-based approaches.

\subsection{Analytical performance bounds}

Characterizing the performance of a wireless network considering only capacity as a performance metric [1113] has triggered a comprehensive study, starting with the seminal work of Gupta and Kumar [14]. Grossglauser and Tse [15] extended the analysis in [14] by considering the impact of mobility on capacity. The authors in $[16,17]$ model information dissemination for the unicast, multicast, and broadcast problems with a focus on capacity.

Analysis of the trade-off between capacity and delay has been investigated by El Gamal et al. in [18]. Comaniciu and Poor [19] accounted for delay as a constraint in their capacity analysis. The compromise between capacity and energy consumption for line networks has been analytically derived by Bae and Stark in [20]. A cross-layer framework for optimal delay-margin, network lifetime and utility tradeoff has been proposed by Tahir and Farrell in [21]. A tight hyperbolic bound on energy and delay for wireless networks has been provided by Brand and Molisch in $[22]$. In $[23,24]$, the trade-off between delay and energy for wireless sensor networks are investigated. In [25], capacity, delay and energy are considered concurrently but analytical bounds are only derived for a reduced line network configuration of nodes in a series formation.

\subsection{Optimization-based derivations}

In Toumpis and Goldsmith [26] associate network design decisions with areas of the performance graphs. The authors' goal is to compare the performance achieved through the implementation of various power, scheduling, queuing, and routing choices with theoretical upper bounds on capacity. The protocols proposed in their work are evaluated in terms of delay vs. throughput and energy per packet vs. throughput. Similarly, the work in [27] studies the impact of power control, rate changes, multi-hop communication, and spatial reuse on the problem of maximizing the minimum throughput. The work later described in [28] then considers the tradeoff between throughput and network lifetime.

Several other works have proposed to derive optimal communication strategies with respect to a specific optimization objective based on the networking application. Previous approaches can generally be classified according to the interaction of considered layers [29]. There are protocol designs based on interactions between Medium Access Control (MAC) and physical layers [30], between network and physical layers [31], etc. In this work, we introduce a cross-layer approach for the design of a multiobjective optimization framework based on the interactions between the network and physical layer. The impact of routing decisions on the interference distribution of the network is fully characterized and hence solutions where interference is minimized can be derived accordingly.

Other approaches to network optimization include constraining the feasible set to account for multiple criteria, 
and/or combining multiple criteria into a single objective function as in [32]. A drawback of the latter approach is the difficulty in tuning the weights of the performance objectives in the composite function. The performance gains and losses are also not obvious when optimizing a composite function or constraining the solution space. For example, the optimization of one objective may result in decreased performance of another objective, but this tradeoff may not be clear. There is an inherent interconnection of performance criteria given the wireless medium. Performance should thus be evaluated and presented in terms of tradeoffs.

It is difficult to derive analytical models that provide bounds for more than two objectives at a time. As a consequence, providing a framework to obtain analytical bounds for multiple optimization objectives becomes relevant in the context of a practical two dimensional network. The modeling framework that we present facilitates the performance evaluation of multiple criteria. For the problem we address, we consider jointly optimizing reliable information transfer, end-to-end delay, and overall energy consumption.

A similar approach in the context of routing design for wireless sensor networks has been proposed in [23]. In this paper, the network is fully connected and the authors look for the set of Pareto optimal routing unicast and multicast paths that minimize energy and delay. Hence, reliability is assumed to be guaranteed since all links are considered perfect and interference-free. This assumption is not made in our case and transmission between any two nodes is modeled using a fading channel. In this paper, the probability of a successful transmission on a channel is derived according to the statistical distribution of interference. Our work therefore introduces a cross-layer approach that accurately accounts for the interference generated by the routing and resource allocation decisions.

Another multiobjective approach for wireless sensor network (WSN) optimization [33] looks to optimize coverage and network lifetime. The authors solve for the nondominated solutions and select the set of active nodes to cover an area specific to a considered application. While the purpose of their study is WSN planning, WSNs is used in our work only to demonstrate our framework. The authors do not consider the impact of transmissions on the layers including interference and scheduling. They perform shortest path routing after the Pareto set is identified for the coverage and lifetime objectives.

The approach of solving for the Pareto set to identify solutions for an application's operating point is shared by the works in [33-35]. Great value exists in solving for the set of performance tradeoffs and identifying the associated network solutions as detailed earlier. Our aim is to provide a multiobjective modeling framework that intersects the research areas identified in [6] for connecting theoretical limits with practical performance. As such, we provide a probabilistic network model relying on a cross-layer approach which intrinsically captures wireless broadcast communications and interference effects. The proposed multiobjective optimization problem captures different routing and resource allocation paradigms while relying on a reduced search complexity compared to other approaches.

\section{A cross-layer framework for network modeling}

\subsection{Probabilistic network model}

A wireless network composed of $N$ nodes is assumed. Our proposed model considers a probabilistic network which is characterized by two probability measures: link and node probability. These two parameters completely characterize the network and capture cross-layer interactions.

The node probability $\left(\chi_{i}\right)$ captures the availability of node $i$ for routing purposes, i.e. the probability that node $i$ re-broadcasts a received packet. The node probability has two components $\left(\chi_{i}=x_{i} \cdot \xi_{i}\right)$, one that is determined by the node's routing choices and called the forwarding probability $x_{i}$, and one component $\xi_{i}$, called the external impact factor, that represents the impact of the environment and protocol implementations at adjacent layers (e.g. congestion models, node failures, security risks, energy levels, etc.). For instance, if nodes fail with a probability $p_{f}$, it may be reflected in $\xi_{i}$ which would be equal to $\left(1-p_{i}\right)$. Similarly, it may be a way to account for decisions made by other layers, such as the medium access (e.g. congestion control mechanisms) or the application layer depending on the context of the application (e.g. security risk management). It is through $\xi_{i}$ that cross-layer interactions including MAC and application layers can be accounted for in our framework. For simplicity we have decided to introduce a unique factor $\xi_{i}$ to represent the impact of all decisions of a node which are not related to routing. Deriving an explicit definition for $\xi_{i}$ based on different assumptions is out of the scope of this paper but will be addressed in future works, as more elaborated networking case studies are analyzed. In our cross-layer framework, we aim to optimize the forwarding probability.

The global link probability $\left(p_{i j}\right)$ captures the link availability, i.e., the probability of a successful transmission over a link $(i, j)$. Characterization of the link probability is impacted by enhancements and impairments at various layers of the protocol stack such as fading at the physical layer or congestion at the MAC layer. Both node and link probabilities are illustrated in Fig. 1.

Node and link probability measures are strongly related due to the broadcast nature of the wireless channel. Hence, once the node probabilities $\chi_{i}$ are set, the activity of every node of the network is fixed and the interference distribution can be completely determined given the activity of the nodes on the wireless channel. As a consequence, the link probabilities can be computed as a function of the signal to interference plus noise ratio (SINR). Once the link and the node probabilities are available, various performance metrics such as delay, reliability, or energy consumption can be calculated for various transmission schemes

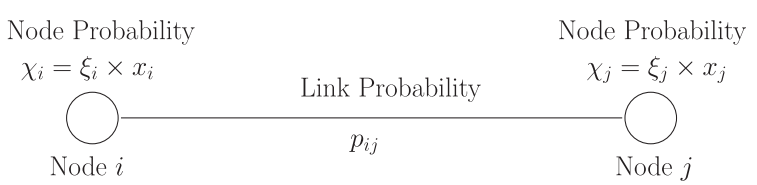

Fig. 1. Node and global link probabilities on a link $(i, j)$. 
(unicast, multicast, broadcast, anycast, etc.). It is important to stress that the proposed cross-layer framework accounts for the interference-limited and broadcast nature of the wireless channel. Indeed, any transmission originating from a node can be received by any other node of the network with probability $p_{i j}$, modeling both the interferencelimited and broadcast features of the wireless channel.

In the following, we consider the set of node probabilities as the variables of the network optimization problem. Finding the best possible routing choices with respect to one particular criterion reduces to the problem of selecting the set of node probabilities that optimizes one particular objective of the network. Within a multiobjective perspective, solving the network optimization problem requires finding the set of Pareto optimal solutions that concurrently optimizes several performance metrics of the network.

To illustrate our framework, we consider here a network where the nodes are independently and randomly distributed according to a random point process of density $\rho$ over a disk $\mathcal{D}$. The communication between any two nodes is performed in a half-duplex mode over a single to multi-hop path. The bandwidth of the channel is divided into $R$ resources (time slots or frequencies). For clarity purposes, we present this model in the context of timemultiplexing.

This paper concentrates on a single flow, but our framework can be extended to multiple flows since the proposed interference model accurately accounts for all the nodes transmitting in the network. Hence, one source transmits a constant traffic in one of the $R$ time slots. We also assume that a relay cannot differentiate packets. As a consequence, all packets are treated as unique by a relay and several copies of a packet can be received at the destination. However, a node relays the packets in the order they are received in one of its available resources. If several packets are received in the same frame, it can only transmit the proportion of packets its forwarding probability $\chi_{i}$ allows. The packets that the node cannot forward are dropped.

\subsection{Link probabilities}

A realistic link $(i, j)$ in time slot $r$ is characterized by its link probability $p_{i j}(r)$, which is a function of the statistical distribution of the SINR at the location of the destination node $j$. Such a computation captures the cross-layer impact of the network routing decision on the physical layer performance since the activity of all the nodes of the network are accounted for statistically in the model. The following are some preliminary definitions and notations that are needed to define the link probability:
Pathloss attenuation factor and transmission power. $a_{i j}$ reflects the attenuation due to propagation effects between nodes $i$ and $j$. In our simulations, the simple isotropic propagation model is considered. We consider that all the nodes use the same transmission power denoted as $P_{T}$.

Interference. Since we consider time-multiplexed channels, interference only occurs between transmissions using the same channel at the same time. Hence, the power of interference $I_{i j}(r)$ on a link $(i, j)$ using resource $r$ and computed at node $j$ is defined by:

$I_{i j}(r)=\sum_{k=1}^{K} P_{T} a_{k j}$ for $k \neq i$

where $K$ is the number of interfering signals in resource $r$.

SINR. The SINR between any two nodes $i$ and $j$ in resource $r$ is given by:

$\gamma_{i j}(r)=\frac{P_{i j}}{N_{0}+I_{i j}(r)}$

where $P_{i j}$ is the power received in $j, I_{i j}(r)$ is the interference power on the link and $N_{0}$ the noise power density. We have $P_{i j}=P_{i} a_{i j}$ for a fixed nominal transmission power $P_{i}$ and a pathloss attenuation factor $a_{i j}$.

Packet error rate (PER). For any value of $\operatorname{SINR} \gamma$, the packet error rate PER can be computed according to:

$$
\operatorname{PER}(\gamma)=1-[1-B E R(\gamma)]^{N_{b}}
$$

where $N_{b}$ is the number of bits of a data packet and $B E R(\gamma)$ is the bit error rate for the specified SINR per bit $\gamma$ which depends on the physical layer technology and the statistics of the channel. Results are given for an AWGN channel and a BPSK modulation without coding where $\operatorname{BER}(\gamma)=Q(\sqrt{2 \gamma})=0.5 * \operatorname{erfc}(\sqrt{\gamma})$.

Frame. The MAC layer consists of frames repeating indefinitely. Each frame is divided into $R$ resources. These $R$ channels can be allocated as time slots or frequencies.

Transmission rate. The activity of a network node in a channel $r \in[1, \ldots, R]$ is given by its transmission rate $\tau_{i}(-$ $r) \in[0,1]$ in that particular channel. This rate is defined as the percentage of time a node $i$ transmits using resource $r$. Fig. 2 shows the set of communication frames each split into $R$ resources. In the case of time slots, node $i$ in the figure sends a packet in time slot $r_{1}$ every three frames for a transmission rate $\tau_{i}(1)=0.33$. Node $j$ transmits every two frames in $r=1$ for a transmission rate of $\tau_{j}(1)=0.5$.

Interfering sets. A node $i$ is said to be active in the network if $\tau_{i}(r)>0$. We define $\mathcal{M}^{r}$ as the set of active transmissions in resource $r$. An interfering set $\mathcal{I}_{i j}^{r}$ for link $(i, j)$ in resource $r$ belongs to the set of all possible interfering sets $\mathcal{L}_{i j}^{r}$. $\mathcal{L}_{i j}^{r}$ is the power set $\mathcal{P}\left(\mathcal{M}^{r}-\{i\}\right)$, i.e. the set of all

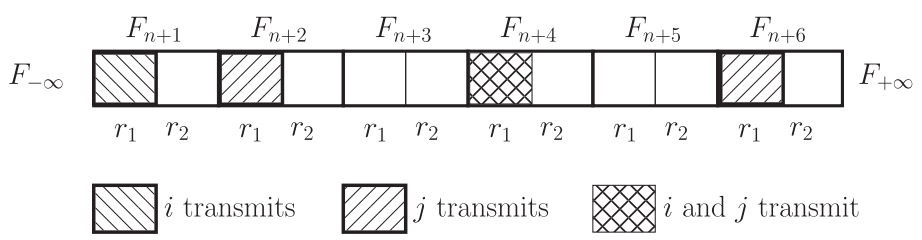

Fig. 2. Frame and transmission rates for the case of $R=2$ and two nodes $i$ and $j$ share slot 1 with $\tau_{i}(1)=0.33$ and $\tau_{j}(1)=0.5$, respectively. 
subsets of $\mathcal{M}^{r}-\{i\}$. Because of the half-duplex constraint, the receiver $j$ is kept in the set $\mathcal{M}^{u}-\{i\}$. Thus, the channel probability computation accounts for the interfering sets where $j$ is active. If $j$ is active, the SINR is very low and transmission on link $(i, j)$ in resource $r$ is impossible. The set $\mathcal{L}_{i j}(r)$ includes the case where no other node is transmitting $(K=0)$ as well.

Link probability. $p_{i j}(r)$ depends on the distribution of the SINR, and consequently on the distribution of the corresponding packet error rates. Eq. (4) details the derivation of $p_{i j}(r)$ as the average of the PER experienced for all possible interfering sets $l \in \mathcal{L}_{i j}(r)$ on resource $r$ referred to as $P E R_{l}$ :

$p_{i j}(r)=\sum_{l \in \mathcal{L}_{i j}(r)}\left[1-P E R_{l}\right] \cdot \mathbf{P}_{l}$

where $P E R_{l}=\operatorname{PER}\left(\gamma_{l}\right), l \in \mathcal{L}_{i j}(r)$. Here $\gamma_{l}$ is the SINR experienced on the link $(i, j)$ in resource $r$ if the nodes of the interfering set $l$ are active.

$\mathbf{P}_{l}$ is the probability for the interfering set $l$ to be active and create interference on the link $(i, j)$ on resource $r$. More specifically, it is the probability that the nodes of the interfering set $l$ are transmitting concurrently and the others are not as specified in the following:

$\mathbf{P}_{l}=\prod_{k=1}^{K} \tau_{k}(r) \cdot \prod_{m=1}^{M-K-1}\left(1-\tau_{m}(r)\right)$

In (5), $\prod_{k=1}^{K} \tau_{k}(r)$ gives the probability that the $K$ active nodes of the interfering set $l$ are transmitting and $\prod_{m=1}^{M-K-1}\left(1-\tau_{m}(r)\right)$ the probability that the $M-K-1$ other active nodes are not.

Global Link probability. For a link $(i, j)$, the global link probability $p_{i j}$ is the probability that a packet arrives with success at node $j$. It is given by:

$p_{i j}=\sum_{r=1 ; \tau_{i}(r) \neq 0}^{R} p_{i j}(r) \frac{\tau_{i}(r)}{\sum_{r} \tau_{i}(r)}$

where $p_{i j}(r)$ is the link probability between $i$ and $j$ for resource $r\left(c f\right.$. Eq. (4)), and $\tau_{i}(r) / \sum_{r} \tau_{i}(r)$ is the probability for the packet to be sent using $r$.

\subsection{Node probabilities and transmission rate}

As we introduced early, the variables of our model are the probabilities $\chi_{i}$ is decomposed into $x_{i}$ and $\xi_{i}$. In the following, we assume $\xi_{i}=1$ to simplify our model for this first investigation. Our model is still inherently cross-layer since it captures the impact of the forwarding and resource allocation decisions at the network and MAC layers on the physical layer by accurately computing the interference distribution in the network. Finally, knowing the optimal routing strategies and corresponding performance trade-offs, a network designer can make informed decisions based on operation and quality of service requirements at the application layer.

There is no explicit notion of routing paths in our model and a packet sent by a source may use one or more paths in parallel to reach the destination, depending on the forwarding decisions of the nodes. For $x_{i}=1$ each received packet by node $i$ is forwarded. For $x_{i}<1$ node $i$ drops the packets with probability $1-x_{i}$. Values of $\left.\left.x_{i} \in\right] 1, R\right]$ are not allowed yet as they imply that node $i$ transmits several copies of the same packet.

As stated earlier, the transmission rate $\tau_{i}(r)$ in resource $r$ is a function of the node probability $x_{i}$ but also depends on the amount of traffic coming into node $i$, which is a function of the activity of the other nodes of the network. As a consequence, computing the values of $\tau_{i}(r)$ knowing the $x_{i}$ values is intractable since determining $\tau_{i}(r)$ requires the knowledge of the link probabilities which are themselves a function of the $\tau_{i}(r)$ values. However, the reverse approach where the variables $x_{i}$ are expressed as a function of the $\tau_{i}(r)$ can be easily derived as stated below. Hence, such a reverse approach leads to the use of the transmission rates as the variables of our multiobjective optimization problem instead of the forwarding probabilities. This reverse approach represents an important contribution of our cross-layer model since it captures an exact picture of the interference distribution at the physical layer and determines the corresponding node forwarding probability $x_{i}$ at the routing level. Fundamental to this derivation is for the flow of interest to be mapped using a directed acyclic graph (DAG) for the considered network topology.

$D A G$ assumption. Assuming a wireless network of $N$ nodes using $R$ resources, a flow between a source $S$ and $D$ and a set of transmission rates $\tau_{i}(r), \forall r \in[1 \ldots R], i \in[1 \ldots N]$, a directed acyclic graph $\mathcal{G}=(V, E)$ where all paths originate at $S$ and end at $D$ is assumed. The set of vertices $V$ is reduced to the set of active nodes $\mathcal{M}$ with nonnull transmission rates on all resources. Oriented edges link any two active nodes with nonnull link probability $p_{i j}$ and belong to a multi-hop path joining $S$ to $D$ without loop. Construction of an instance of this graph is out of the scope of this paper and will be investigated in later studies.

For any node $i \in V$, we denote by $\mathcal{V}_{i}^{\text {in }}$ the set of incoming oriented edges coming from its previous hop neighbors. Similarly, $\mathcal{V}_{i}^{\text {out }}$ denotes the set of outgoing edges to any next hop neighbor node. The set of previous hop neighbors $\mathcal{N}_{i}^{\text {in }}$ and next hop neighbors $\mathcal{N}_{i}^{\text {out }}$ of $i$ is defined as well: $\mathcal{N}_{i}^{\text {in }}=\{k \in V:(k, i) \in E\}$ and $\mathcal{N}_{i}^{\text {out }}=\{j \in V:(i, j) \in E\}$.

Relationship between $x_{i}$ and the $\tau_{i}(r)$. Given the values of $\tau_{j}(r), \forall j \in \mathcal{N}_{i}^{i n}$, we can define the quantity of information coming from all the previous hop neighbors of node $i$ by:

$q_{i}=\sum_{k \in \mathcal{N}_{i}^{\text {in }}} \sum_{r=1}^{R} p_{k i}(r) \cdot \tau_{k}(r)$

where $p_{k i}(r) \cdot \tau_{k}(r)$ is the probability that a packet arrives in node $i$ from previous hop node $k$ in resource $r$.

The quantity of information going out of $i$ is given by the sum of the $\tau_{i}(r)$ over all the time slots. Hence, we can determine the forwarding probability of $i$ to be:

$x_{i}=\frac{\sum_{r} \tau_{i}(r)}{\sum_{k \in \mathcal{N}_{i}^{\text {in }}} \sum_{r=1}^{R} p_{k i}(r) \cdot \tau_{k}(r)}$

Main variables and parameters are summarized in Table 1 for the reader's convenience.

\section{A multiobjective optimization problem}

The performance of most wireless networks can be assessed with regards to various criteria such as throughput 
or capacity, end-to-end transmission delay, overall energy consumption or transmission reliability. The purpose of the multiobjective framework presented in this work is to determine, given a network and a communication pattern, what kind of trade-offs arise between chosen performance metrics when varying the routing strategies. It relies on the cross-layer probabilistic network model presented in Section 3.

\subsection{Variables of the multiobjective (MO) framework}

The routing strategies are the variables of our multiobjective optimization problem and a solution is defined by:

Definition 1. A solution $\mathcal{S}$ of the MO framework is defined by the set of transmission rates $\tau_{i}(r) \in[0,1]$ used by each node $i$ on each resource $r$ :

$\mathcal{S}=\left\{\tau_{i}(r)\right\}_{i \in[1, \ldots, N], r \in[1 \ldots R]}$

The set of forwarding probabilities $x_{i, i \in[1 \ldots N]}$ is derived according to Eq. (8) and represents the routing strategy of the network. Each variable $\tau_{i}(r)$ takes its values in a discrete set $\Gamma$ of size $T=|\Gamma|$. As a consequence, the solution space is derived as:

$|\mathcal{S}|=\sum_{m=0}^{(N-2)}\left(\begin{array}{c}N-2 \\ m\end{array}\right) T^{R \cdot m}$

In order to reduce the size of this very big search space, we only consider solutions where at least one cumulative time slot per node is available in the frame, i.e.

s.t. $\forall i \in[1, N], \sum_{r=1}^{R} \tau_{i}(r) \leqslant R-1$. The solutions that do not meet this constraint are usually very bad solutions since at least one of the nodes of the solution is transmitting in all its time slots preventing a failure free packet reception.

Using this definition of a routing strategy, a solution may reflect various routing strategies: it can be single-hop or multi-hop, single path or multi-path, probabilistic or deterministic.

\subsection{MO-Tabu: a multiobjective optimization heuristic}

The aim of our MO framework is to obtain the set of Pareto optimal routing strategies of the MO problem. A Pareto optimal set is composed of all the non-dominated solutions of the MO problem with respect to the performance metrics considered. The definition of dominance is:

Definition 2. A solution $A$ dominates a solution $B$ for a $n$-objective MO problem if $A$ is at least as good as $B$ for all the objectives and $A$ is strictly better than $B$ for at least one objective. Mathematically, we have for a minimization problem:

$\forall i \in[1, n]: f_{i}(A) \leqslant f_{i}(B), \exists j \in[1, n]: f_{j}(A)<f_{j}(B)$

The considered optimization problem is solved using a multiobjective metaheuristic called PMOTS (Parallel MultiObjective Tabu Search) described in [9]. It is based on the Tabu metaheuristic [36], a local search using a list of Tabu solutions to reduce the occurrence of loops in the search.
PMOTS is a multiobjective extension of Tabu search where $K$ Tabu searches are performed in parallel. The goal of this algorithm is to obtain the best possible approximation of the Pareto optimal set of solutions $F_{P}$. A more detailed description may be found in Appendix A.

\section{A first application to sensor networks}

We propose in the following to assess the performance of a wireless sensor network (WSN) by capturing the tradeoffs that arise between end-to-end reliability, overall energy consumption, and end-to-end delay. These criteria are most relevant since providing a maximal network throughput is usually not the main task of a WSN.

\subsection{Maximum number of hops}

The following criteria are defined for a single sourcedestination pair $(S, D)$ and for a fixed maximum number of hops $H_{M}$ a packet can travel in the network. The quantity $H_{M}$ is similar to a Time To Live (TTL) tag appended to a packet transmitted in the network. Consequently, the optimization criteria will account for the performance of all possible paths between $S$ and $D$ which are composed of at most $H_{M}$ hops.

Since the model we have defined in Section 3 relies on a DAG representation of the network topology for the flow of interest, the maximum length of paths in the network is finite but can be higher than our fixed limit $H_{M}$. As such, we introduce a binary variable $v_{k i}$ to represent the usefulness of the link $(k, i)$ with respect to the maximum number of hops constraint. Hence, if link $(k, i)$ does not belong to a path of length $H_{M}$ hops between $S$ and $D$, we have $v_{k i}=0$. On the contrary, we have $v_{k i}=1$ if $k$ only receives packets with number of hops $h<H_{M}$, meaning it belongs to at least one path of $H_{M}$ hops between $S$ and $D$.

Setting $H_{M}$ impacts the definition of $x_{i}$ in (8). Eq. (8) is modified as follows:

$x_{i}=\frac{\sum_{r} \tau_{i}(r)}{\sum_{k \in \mathcal{N}_{i}^{\text {in }}} \sum_{r=1}^{R} p_{k i}(r) \cdot \tau_{k}(r) \cdot v_{k i}}$

\subsection{Reliability criterion}

Reliability is defined as the probability that a packet emitted at $S$ successfully arrives at $D$ in at most $H_{M}$ hops. The reliability criterion is given by:

$f_{R}=\mathcal{P}\left(T_{S D}^{H_{M}}\right)$

For any two nodes $i$ and $j$ of the network, $T_{i j}^{H}$ represents the event that a packet transmitted by $i$ successfully arrives at $j$ in at most $H$ hops. Our aim is to maximize $\mathcal{P}\left(T_{S D}^{H_{M}}\right)$ which is defined as follows.

Definition 3. $\mathcal{P}\left(T_{S D}^{H_{M}}\right)$ is the probability that the packet arrives successfully at $D$ in at most $H_{M}$ hops and is given by:

$\mathcal{P}\left(T_{S D}^{H_{M}}\right)=1-\prod_{h=1}^{H_{M}}\left(1-\mathcal{P}\left(T_{S D} \mid H=h\right)\right)$ 
where $\mathcal{P}\left(T_{S D} \mid H=h\right)$ is the probability for a packet to arrive in $h$ hops at $D$. For $h=1, \mathcal{P}\left(T_{S D} \mid H=1\right)=p_{S D}$, the successful transmission probability on the link $(S, D)$ following Eq. (6). For $h>1$, we have:

$\mathcal{P}\left(T_{S D} \mid H=h\right)=1-\prod_{j=1}^{\mathcal{N}_{S}^{\text {out }}}\left[1-p_{S j} x_{j} \mathcal{P}\left(T_{j D} \mid H=h-1\right)\right]$

with $\mathcal{N}_{S}^{\text {out }}$ the number of possible next hop relays of $S$; $p_{S j}$ the link probability between $S$ and its neighbor $j$; $\mathcal{P}\left(T_{j D} \mid H=h-1\right)$ the probability to reach $D$ in $(h-1)$ hops and $x_{j}$ the forwarding probability of $j$.

To reduce the computational complexity of the reliability probability, a restricted set $\mathcal{N}_{S}^{\text {out }}$ of next hop relays may be considered but the loss in terms of accuracy is hard to quantify. Therefore, we rather introduce a link threshold value $\mathcal{P}_{\text {th }}$ computed for each path made of $h$ hops. While recursively calculating $\mathcal{P}\left(T_{S D} \mid H=h\right)$, if the probability of a path gets lower than $\mathcal{P}_{t h}$, the recursion is stopped for that particular path and its contribution to $\mathcal{P}\left(T_{S D} \mid H=h\right)$ is set to zero.

\subsection{Delay criterion}

The end-to-end delay is the sum of the times spent at each relay on a multi-hop path [18] because all nodes transmit at the same $1 \mathrm{Mbps}$ rate and propagation delay is negligible. We assume that there is a finite bound on the time a packet spends in the outgoing buffer of any node of the network. In this case, each relay introduces a normalized delay of one on the end-to-end delay. The criterion $f_{D}$ is defined by:

$f_{D}=R \cdot \sqrt{\sum_{h=1}^{H_{M}}(h-1)^{2} \cdot R_{h}}$

The quantity $(h-1)$ is the delay needed by a packet to arrive in $h$ hops using $(h-1)$ relay nodes. The scaling factor $R$ represents the delay induced by the $R$ resources. $R_{h}$ is the probability that the packet arrived in exactly $h$ hops and did not arrive in one, two, or $(h-1)$ hops. For $h=1$, we have $R_{h}=P\left(T_{S D} \mid H=1\right)$ and for $h>1$ :

$R_{h}=\mathcal{P}\left(T_{S D} \mid H=h\right) \cdot \prod_{i=1}^{h-1}\left(1-\mathcal{P}\left(T_{S D} \mid H=i\right)\right)$

If no route exists between $S$ and $D$ then $f_{D}=+\infty$.

\subsection{Energy criterion}

The energy criterion $f_{E}$ is given by the total forwarding energy needed for a packet sent by $S$ to reach $D$. We do not account for the energy spent by the initial transmission in $S$. The reception (respectively transmission) of a packet at node $j$ in resource $r$ consumes $e_{j}^{R}(r)$ (resp. $e_{j}^{T}(r)$ ). Hence, the energy criterion is defined as:

$f_{E}=\sum_{h=1}^{H_{M}} \mathcal{E}\left(T_{S D} \mid H=h\right)$

where $\mathcal{E}\left(T_{S D} \mid H=h\right)$ is the total energy needed by the $h$-hop communications between $S$ and $D$ defined by:

$$
\begin{aligned}
\mathcal{E}\left(T_{S D} \mid H\right. & =h) \\
& =\sum_{j=1}^{\mathcal{N}_{S}^{\text {out }}}\left(p_{S j} \cdot e_{j}^{R}+p_{S j} \cdot x_{j} \cdot\left[e_{j}^{T}+\mathcal{E}\left(T_{j D} \mid H=h-1\right)\right]\right)
\end{aligned}
$$

In Eq. (19), $p_{S j} \cdot e_{j}^{R}$ is the energy consumed for a packet reception by the neighbor $j$ of $S ; p_{S j} \cdot x_{j} \cdot e_{j}^{T}$ is the energy consumed for the packet transmitted by neighbor $j$ and $p_{S j} \cdot x_{j} \cdot \mathcal{E}\left(T_{j D} \mid H=h-1\right)$ is the total energy consumed by the remaining possible paths made of $(h-1)$ hops between neighbor $j$ and the destination. For $h=1$, $\mathcal{E}\left(T_{S D} \mid H=1\right)=0$ since the energy in $S$ is not accounted for.

\section{Results for a single source-destination pair}

\subsection{M-Relay problem}

The results presented in this section are obtained for a small problem instance for three reasons. First, we are able to determine the whole Pareto optimal set of solutions using an exhaustive search. Secondly, such a problem can be easily analyzed and provides a first illustration of our multiobjective framework. Lastly, it is used in Appendix B to assess the efficiency of the multiobjective optimization metaheuristic we developed to tackle bigger problem instances [9].

In the following, the network is composed of $N=333$ nodes uniformly distributed with density $\rho=0.004$ over a disk $\mathcal{D}$ of radius $R_{\mathcal{D}}$. The distance between $S$ and $D$ is about $215 \mathrm{~m}$. To reduce border effects, $S$ and $D$ are selected within a radius $R_{\mathcal{C}} \ll R_{\mathcal{D}}$ which ensures that the power of a node at distance $R_{\mathcal{C}}$ is below the noise power for the nodes located at distance $R_{\mathcal{D}}$. We consider $R=2$ time slots and use a probabilistic discrete variable space. A link reliability threshold of $\mathcal{P}_{t h}=10^{-10}$ is set. Propagation and physical layer parameters are summarized in Fig. 5. Energy consumption for emission and reception of packet of size 5000 bits for the type of sensors considered in [37] is assumed. Main energy is consumed by packet emission here.

The dimension of the search space can be modified by setting a maximum number of forwarding nodes $M$ in a solution $\mathcal{S}$. This sub-problem is addressed in the following as the $M$-relay problem instance and has a reduced search space compared to the original one of Eq. (10). To compute the complexity of this search space, we assume that at most $M$ relays compose a solution, $R=2$ time slots exist and that the constraint:

$\forall i \in[1, N], \quad \sum_{r=1}^{R} \tau_{i}(r) \leqslant R-1$

is realized to permit packet reception in at least one slot out of two. Complexity of the M-relay problem with $R=2$ is derived as follows:

$|\mathcal{S}|=\sum_{m=0}^{M}\left(\begin{array}{c}N-2 \\ m\end{array}\right)\left(\frac{(T-1)(T+2)}{2}\right)^{m}$

It sums the set of solutions composed of $0,1, \ldots, M$ relays. For solutions composed of $m \leqslant M$ relays, the constraint of 
Eq. (20) imposes that for one relay of a $m$ - relay solution, there are $(T-1)(T+2) / 2$ possible time slot allocations. All combinations of selecting $m$ relays among $N-2$ are accounted for with the factor $\left(\begin{array}{c}N-2 \\ m\end{array}\right)$.

The cardinality of the M-relay problem is mainly reduced because of the limited summation over $m$. It is clear that the proposed approach will not scale to account for solutions with a very large $M$. However, since we are looking for compromises with reduced energy, delay and high capacity, it is beneficial to search for solutions with a small number $M$ of relays. As we will show in the rest of this section, this assumption is not limiting the characterization of the Pareto optimal performance limits of small to medium scale networks.

In order to cope with large scale networks, other approaches considering continuous network models [38] or mean field theory [39] are more appropriate. The downside of these approaches is that the granularity of the problem definition does not permit the derivation of exact Paretooptimal performance and we lose the complete description of the Pareto-optimal set.

\subsection{Pareto optimal set for the 1-relay problem}

In this problem instance, we set $M=1$ and $H_{M}=2 . \tau_{i}(r)$ takes values in the set $\Gamma=\{0,0.05,0.1, \ldots, 0.9,0.95,1.0\}$ of $|\Gamma|=21$ elements. In that particular case, the search space has a dimension of 76,131 solutions and the Pareto optimal set is obtained with an exhaustive search.

For this instance, the direct link $(S, D)$ is very weak. A reliability of only $\mathcal{P}\left(T_{S D}^{H_{M}}\right)=0.0003$ is achieved with a delay of $f_{D}=0$ and an energy of $f_{E}=0$. Only 24,820 solutions fulfill the constraint $x_{i} \leqslant 1$ that forbids a node to duplicate packets. Among these solutions, 3855 solutions are Pareto optimal, representing about $5 \%$ and $15 \%$ respectively of the whole and the constrained solution space. For all the Pareto optimal solutions the relay never transmits in the first time slot concurrently with the source. The performance of the Pareto optimal set of solutions is represented in Fig. 3 in the space defined by the three evaluation functions. For clarity purposes, the projections of the Pareto set on the reliability-delay, reliability-energy, and the delay-energy planes are also displayed in Fig. 4. The plots show that an improved reliability is obtained at the price of an increase in delay and energy. The trade-off between reliability and delay can be easily understood since higher reliability is achieved when the relay contributes with a higher forwarding probability $x_{i}$, inducing an increase in delay. Similarly, an increase of $x_{i}$ triggers an increased average energy consumption since the relay is forwarding packets more often.

The Pareto set is composed of solutions for which relays belong to a set of 226 nodes, which represents about two thirds of the number of nodes of the network. The location in the network of these 226 nodes is presented in Fig. 6. We also highlighted on this figure the relays that provide a near perfect transmission. We can conclude that the relays located in an ellipse near the middle of the $(S, D)$ distance provide the best reliability at the price of the highest delay and energy. The other relays present in the Pareto set provide various trade-offs depending on their values of $\tau_{i}(r)$.

The Pareto optimal solutions composed of the same selected relay are represented in the objective space in Fig. 7. Three sets of solutions are represented corresponding to three different relays. The locations of the selected relays are also highlighted in Fig. 7. All solutions related to the same relay create a line in the 3-D space defined by the three optimization criteria. Each point of this line is obtained for the same relay but for a different value of $\tau(2)$ (as stated earlier, there is no solution with a relay transmitting on $\tau(1)$ in the Pareto optimal set). For one particular relay, there are at most 21 values, corresponding to the 21 values of $\tau_{i}(r)$ defined in this problem instance. There are relays that provide less than 21 Pareto optimal solutions because some values of $\tau_{i}(2)$ were not valid considering the $x_{i} \leqslant 1$ constraint. It can be clearly seen that relay 103 is one of the relays that provide the highest possible reliability. In this specific example, the location of the relay conditions the maximum achievable reliability and the choice of its transmission rate $\tau_{i}(2)$ modifies the energy versus delay trade-off.

A continuous transmission rate variable $\tau_{i}(r)$ would provide continuous lines instead of discrete points. However, tackling the continuous formulation of our problem is much more challenging and for our study, we will stick to the simpler discrete formulation which still provides a fair representation of the Pareto set.

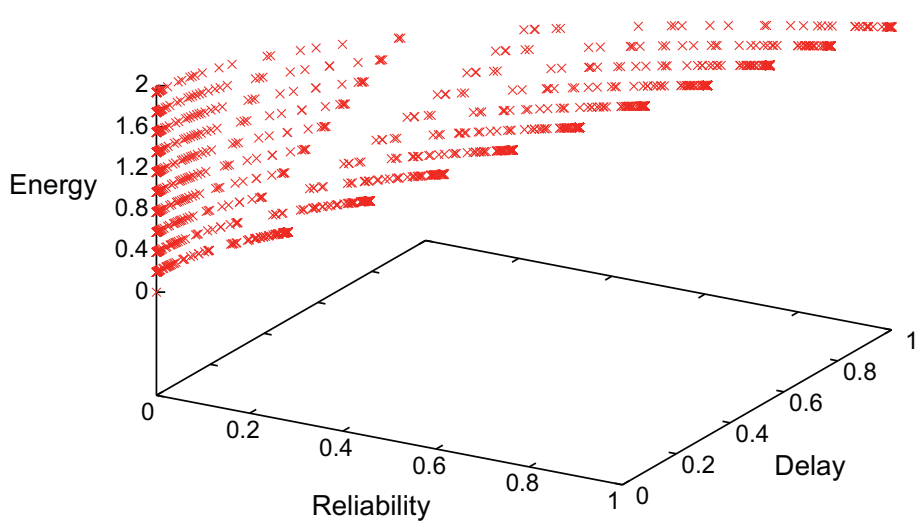

Fig. 3. 3D plot of the Pareto optimal set for the 1-relay problem. 

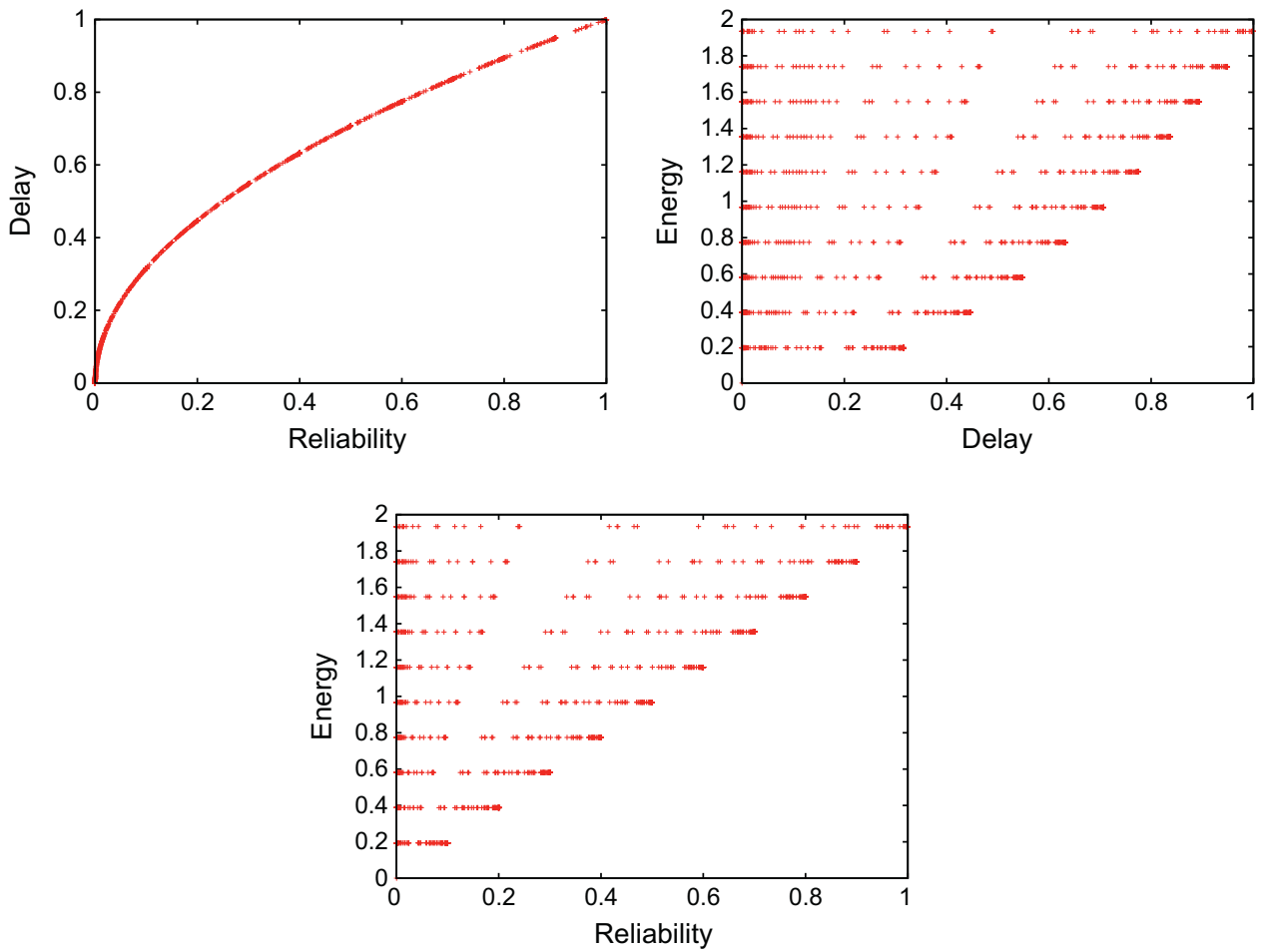

Fig. 4. Representation of the projections of the Pareto optimal set for the 1-relay problem.

\begin{tabular}{|c|c||c|c|}
\hline Transmission Power & $151 \mathrm{~mW}$ & $N_{0}$ & $-154 \mathrm{dBm} / \mathrm{Hz}$ \\
\hline Bandwidth & $1 \mathrm{Mbps}$ & $f$ & $2.4 G H z$ \\
\hline Pathloss exponent $\alpha$ & 3 & Channel Model & AWGN \\
\hline Antenna gains & $G_{T}=G_{R}=1$ & Modulation & BPSK \\
\hline Emission energy & $e^{S}=1.93 \mathrm{~J}$ & Reception energy & $e^{R}=0.74 \mathrm{~mJ}$ \\
\hline
\end{tabular}

Fig. 5. Propagation and physical layer parameter values from [37].

\subsection{Pareto optimal set for the 2-relay problem}

In this problem instance, we set $M=2$ to allow solutions of either one or two active relays. We set $H_{M}=3$, meaning that we account for all the paths having $h \leqslant 3$ hops in the criteria computation. The granularity of the $\tau_{i}(r)$ variable is reduced, and it takes its values in the set $\Gamma=\{0,0.1, \ldots, 0.9,1.0\}$ of $|\Gamma|=11$ elements. The search space has a cardinality of $230,769,891$ solutions. An estimate of the Pareto optimal set is presented in Fig. 8 after 6100 iterations and is composed of 75,577 solutions. On the reliability-energy projection and on the delay-energy projection, we have highlighted the solutions composed of only one relay using a red cross marker.

As shown in the projections of Fig. 8, the same tradeoffs between reliability and delay exist as in the 1-relay subproblem. The shapes of the other plots also resemble the 1-relay subproblem. As in the 1-relay case, most of the solutions in the front are divided into various energy levels because of the discretization of the $\tau_{i}(r)$ space.
Fig. 9 displays the nodes involved in solutions with two active relays. The nodes present in the solution set of the 2relay problem, but not in the 1-relay problem are highlighted with purple squares. They are concentrated at the edge of the network near the source and destination. Solutions which provide a high reliability $\left(f_{R}>0.999\right)$ using two active nodes are denoted with a black circle marker. Most of the solutions providing a quasi perfect reliability are composed of a single relay. With this particular configuration and network, it makes sense since intuitively the use of a single relay is the best possible configuration to mitigate interference for a 2-time slot system. For the highly reliable solutions which have two nodes participating, one relay node is located 'behind' the source and the second relay is located between the source and destination. In this case it is the centrally located node that is contributing the most to the reliability criterion.

For energy constrained wireless networks, it is interesting to see which solutions provide the best possible compromise between energy and reliability. It is clear from 


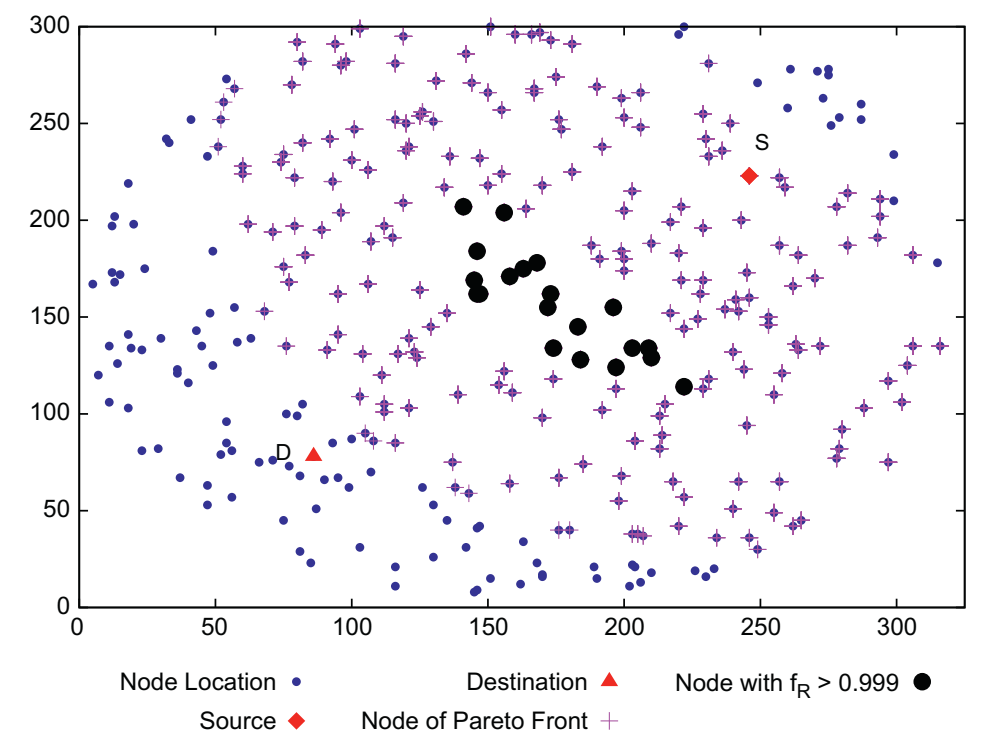

Fig. 6. 1-Relay problem: Location of the nodes that provide Pareto optimal solutions (purple crosses) and of the nodes that provide a near quasi-perfect reliability (full black dots), i.e. $f_{R}>0.999$. (For interpretation of the references to colour in this figure legend, the reader is referred to the web version of this article.)

Fig. 8 that such solutions are composed of a single relay located in between $S$ and $D$ (cf. Fig. 6). For such 1-relay solutions, optimum reliability-energy trade-off is of $\left(f_{R}=1\right.$, $f_{E}=2$ ). Looking at the solutions of Fig. 8 composed of two relays only, the solutions with an energy criterion $f_{E} \leqslant 2$ have a maximum reliability of a little more than 0.45 . The relays locations of the three solutions with $f_{R}>0.45$ and $f_{E} \leqslant 2$ are represented in Fig. 10. For these three solutions, one of the relays is only active in the first time slot with $\tau(1)=0.1$ while the other one is only active in the second time slot with $\tau(2)=0.5$. All three solutions have a reliability very close to 0.45 . Lost packets are due to interference between source and relays transmitting in the same time slot one. It is clear from this study that 2-relay solutions are not of any use for this network if a low energy and high reliability is targeted.

This first simple study shows that the proposed multiobjective probabilistic network model provides a coherent and complete view of the trade-offs that arise between reliability, delay, and energy in our network. For larger networks or when more relays are needed, our problem is solved using the multiobjective optimization algorithm PMOTS as presented in Section 4.2. Although this paper does not concentrate on the description and the performance analysis of PMOTS, we highlight the convergence properties of the algorithm for the 1-relay and 2-relay problems in Appendix B.

\subsection{Model assessment through simulations}

The previously derived Pareto fronts for the 1-relay and 2-relay problems are assessed through simulations completed with the WSNet simulator [40]. WSNet is an event-driven simulator tailored for wireless sensor networks. Additional to common event-driven wireless network simulators, it features detailed propagation models and energy monitoring capabilities of sensor devices. Each Pareto-optimal solution is simulated using the same parameters as used for Fig. 5 and considering the same network distribution as described in Section 6.1. The basic forwarding procedure of RELAY described in Algorithm 1 is implemented.

\section{Algorithm 1. RELAY algorithm}

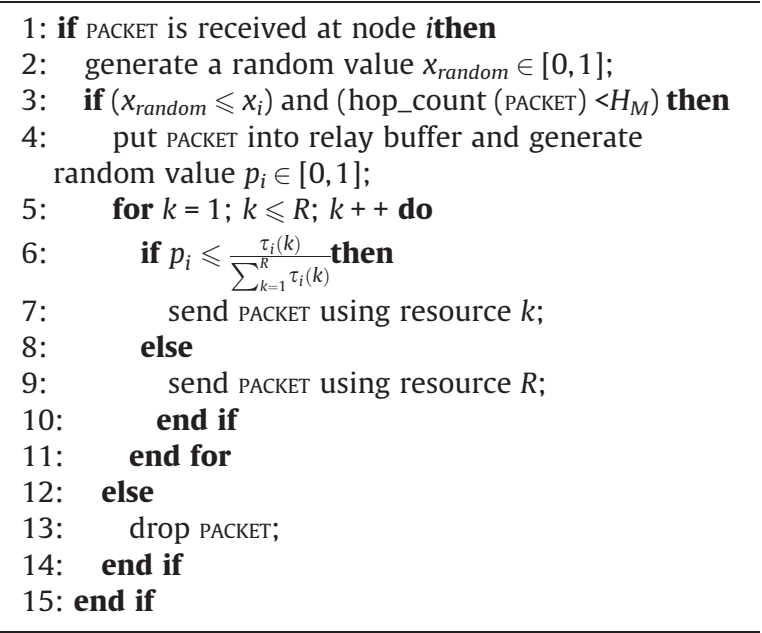

Sources transmit $N_{S}=10,000$ packets before performance criteria are computed as follow:

Reliability. The end-to-end reliability corresponds to the average number of packets successfully received at $D$ in at most $H_{M}$ hops:

$\tilde{f}_{R}=\frac{\sum_{i=1}^{N_{S}} \mathbb{1}_{\text {packet } i \text { received in } D]}}{N_{S}}$ 


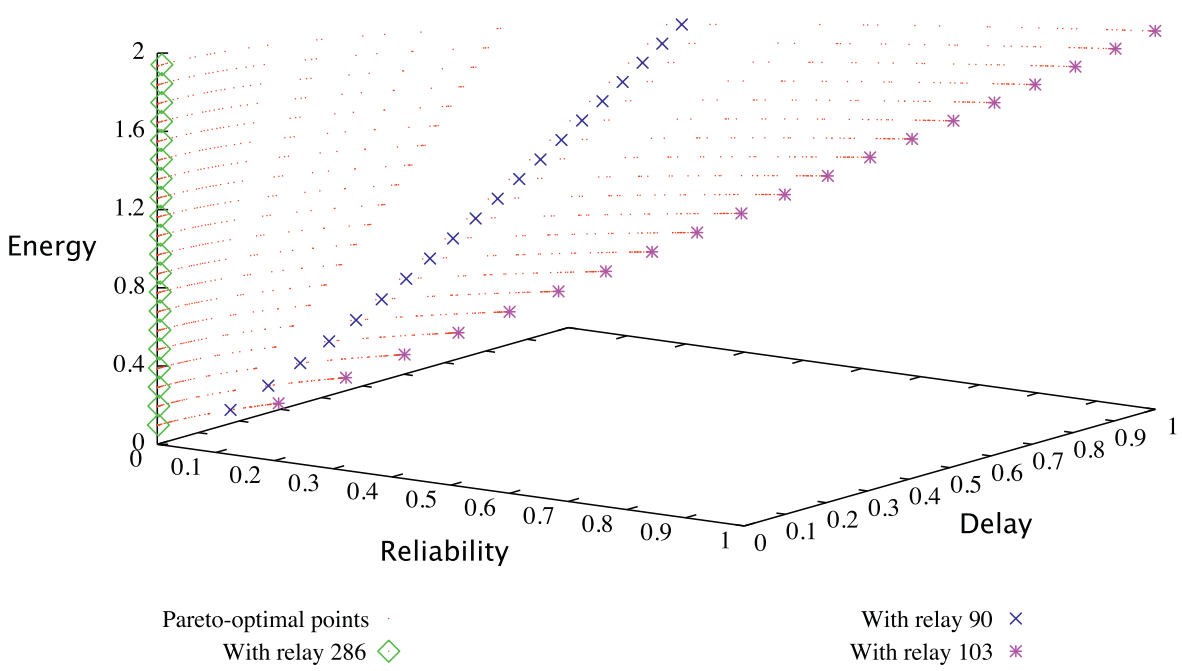

(a)

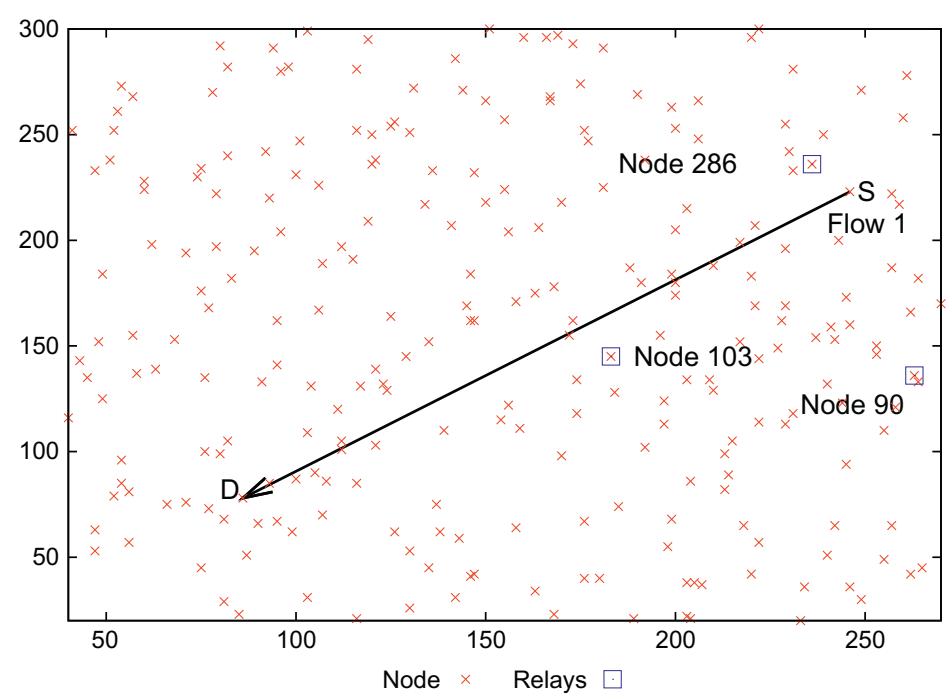

(b)

Fig. 7. 1-Relay problem: Selection of the Pareto optimal solutions using a same relay.

Delay. The end-to-end delay is measured in three steps as follows. Firstly, the total number of packets $n(h)$ arrived in $h$ hops at $D$ is counted for $h \leqslant H_{M}$. Secondly, the probability $R_{h}$ that the packet arrived in exactly $h$ hops is computed using $R_{h}=\frac{n(h)}{N_{\varsigma}}$. Lastly, the delay criterion obtained by simulations $\tilde{f}_{D}$ is derived based on the following formulation:

$\tilde{f}_{D}=\sqrt{\sum_{h=1}^{H_{M}}(h-1)^{2} \cdot R_{h}}$

Energy. The energy consumption is measured by counting the total number of packets received $t_{r}$ and sent $t_{s}$ in the network for the whole simulation duration. Simulated energy criterion is then derived according to:

$\tilde{f}_{E}=\frac{t_{r} e^{R}+t_{S} e^{S}}{N_{S}}$ where $e^{R}$ and $e^{S}$ denote energy consumption of packet reception and emission, respectively Fig. 5.

In order to measure the differences between each criteria predicted by the model and evaluated by WSNet, we adopt a normalized root-mean-square error (RMSE) metric. Normalized RMSE can be computed as:

$\operatorname{RMSE}(f, \tilde{f})=\frac{\sqrt{\sum_{i=1}^{N} \frac{[f(i)-\tilde{f}(i)]^{2}}{f(i)^{2}}}}{N}$

where $f$ and $\tilde{f}$ are the criteria derived from the MO model and from the WSNet simulations, respectively. $N$ is the total number of solutions compared. Table 2 presents the RMSE values for the 1 and 2-relay Pareto sets presented earlier. Relative RMSE is really low, showing that for these networks the model accurately estimates reliability, delay and energy criteria. 

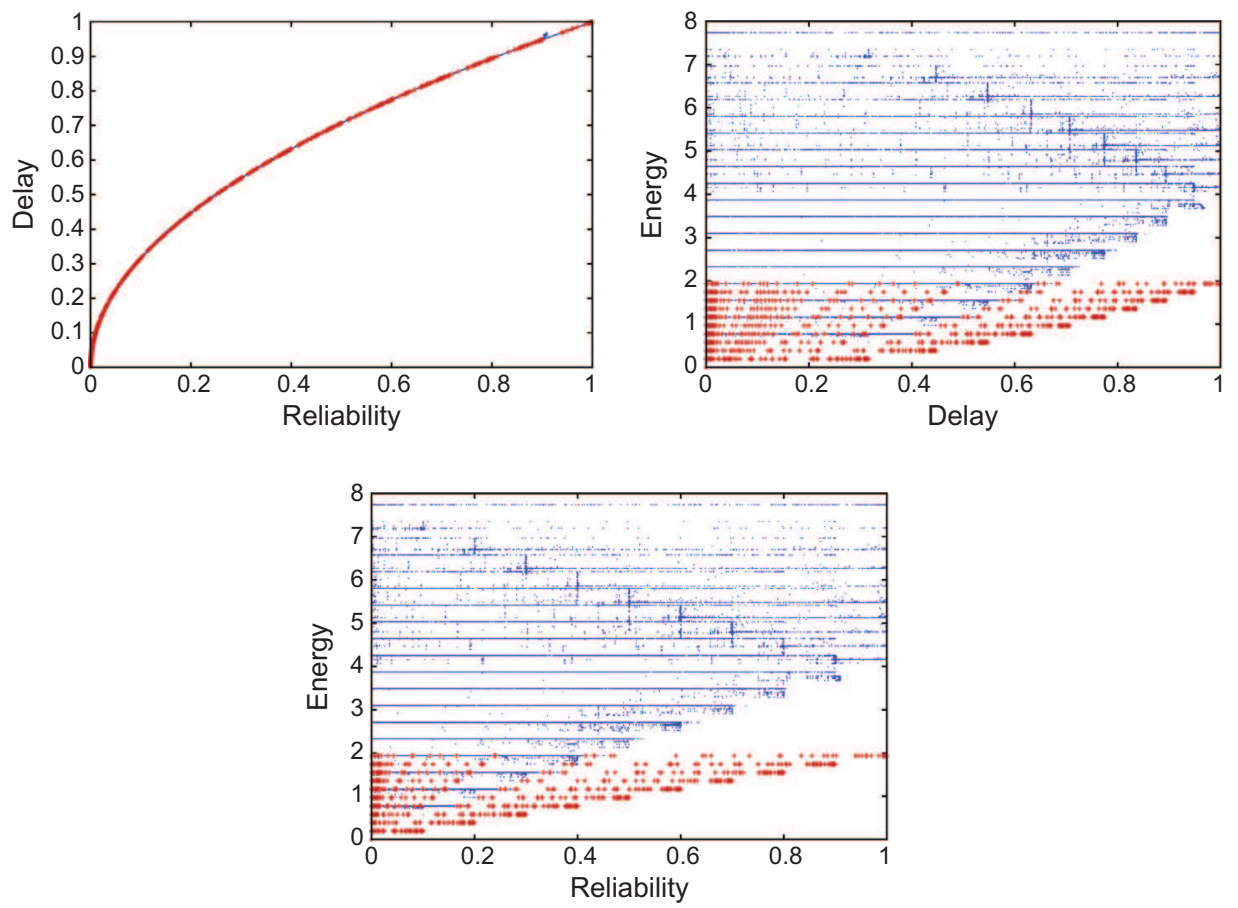

Fig. 8. Projections of the Pareto optimal set for the 2-relay problem. The solutions are divided into two sets: the set of 2682 solutions using only one relay (red cross marker) and the set of 72,895 solutions using two relays (blue dot marker). (For interpretation of the references to colour in this figure legend, the reader is referred to the web version of this article.)

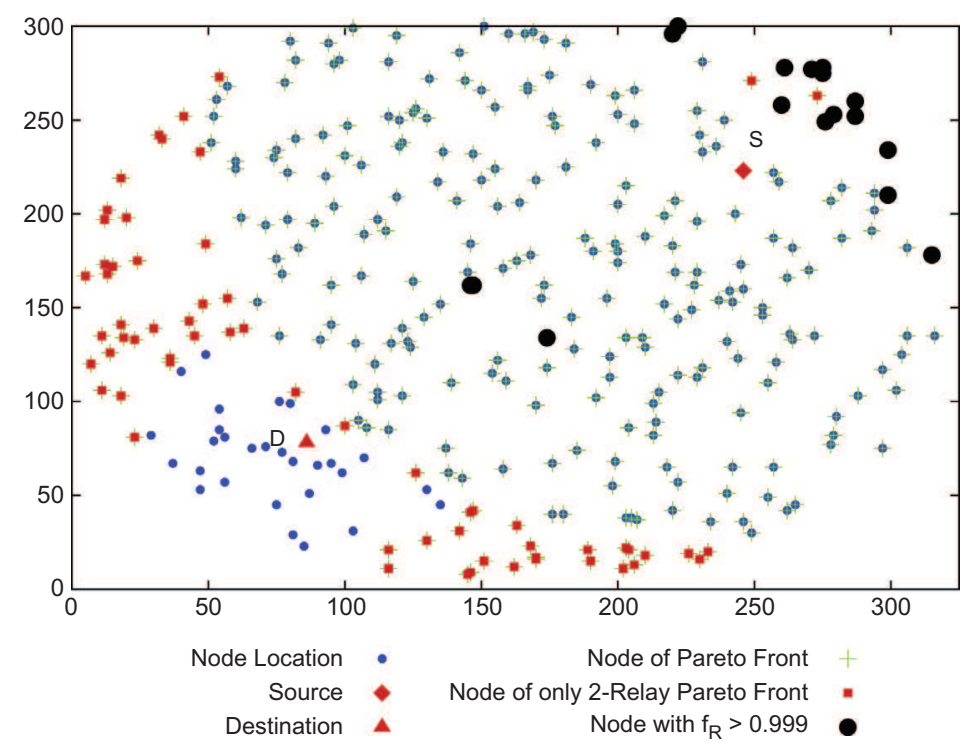

Fig. 9. 2-Relay problem: location of the nodes that provide Pareto optimal solutions (blue crosses) and of the nodes that provide a near quasi-perfect reliability (full black dots), i.e. $f_{R}>0.999$. Nodes present in the Pareto optimal set for the 2-relay problem but not for the 1-relay problem are highlighted with purple square markers. (For interpretation of the references to colour in this figure legend, the reader is referred to the web version of this article.)

\subsection{Impact of the geometry of the network}

In this section, we investigate the impact of various network parameters on the Pareto optimal front. We consider the 1-relay problem to obtain several Pareto optimal fronts for our study with a limited computational time and draw a first set of conclusions. In the following, we still consider a network of randomly distributed nodes of density $\rho$. Three parameters can be modified in the network definition: (i) the location of the relays in the network, (ii) the 


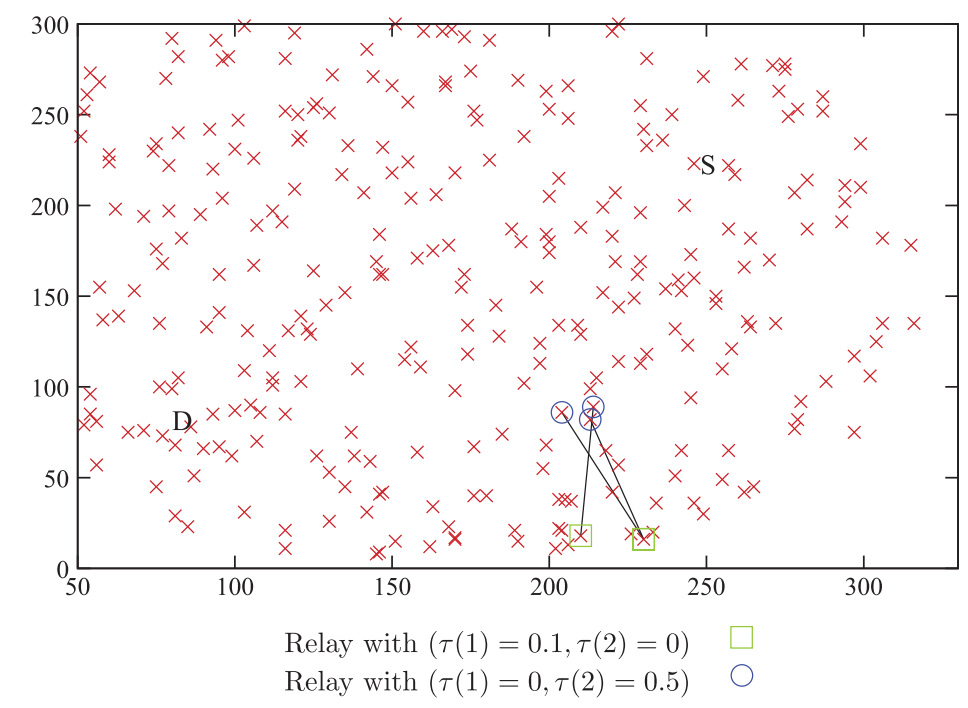

Fig. 10. 2-Relay problem: Location of nodes composing 2-relay solutions with $f_{R}>0.45$ and $f_{E} \leqslant 2$.

network density, and (iii) the geometry of the source-destination flow.

Impact of the location of the relays. The impact of the location of the relay nodes on the Pareto optimal set of solutions is studied. All network instances presented here are obtained for the same node density of $\rho_{0}=0.004$ and the same source destination flow ( $c f$. flow 1 in Fig. 14). The Pareto optimal set of solutions is obtained using an exhaustive search for five network instances. The fronts are presented in Fig. 11. To better see the distribution of the solutions on the Pareto front, Fig. 12 zooms on the highly reliable solutions.

From Fig. 11, it can be observed that all fronts overlap but are different. They all belong to the same surface but provide a different discretization of it. Depending on its location, a relay provides a different line of trade-off values on the Pareto optimal front as shown earlier on Fig. 7. Each different network instance provides another set of possible relays but the same trade-offs are observed between reliability, energy and delay for each of them.

Impact of the network density. The impact of the network density is investigated in the following. The same source destination flow 1 is considered and two Pareto optimal fronts are computed with a density of $\rho=2 \rho_{0}$ and $\rho=6 \rho_{0}$, with $\rho_{0}=0.004$ the density of the original network of Fig. 11. It can be seen on the plots of Fig. 13 that with more nodes present in the network, the Pareto optimal set gets more dense. Adding relays gives a more precise description of the Pareto optimal set. The complete set would be obtained for a continuous definition of the
Table 2

RMSE for reliability, delay and energy.

\begin{tabular}{llll}
\hline Network & RMSE for $f_{R}(\%)$ & RMSE for $f_{D}(\%)$ & RMSE for $f_{E}(\%)$ \\
\hline 1-Relay & 0.31 & 0.57 & 0.08 \\
2-Relay & 0.06 & 0.09 & 0.007 \\
\hline
\end{tabular}

problem where both the locations of the relays would be continuous and the values of the transmission rates. Our current approach does not permit a continuous definition of the network and its parameters. In this context, the novel works on massively dense networks $[41,38]$ provide an appropriate framework for pursuing an extended analysis based on this paper and deriving an asymptotic description of the Pareto optimal sets.

For the 1-relay problem, the density of the network does not affect the geometry of the Pareto optimal set. However, this may not be the case for the $M$ - relay problems with $M>1$. Indeed, increasing the number of potential relays may provide different multi-hop transmission strategies with better performance. However, we conjecture that once a limit density is reached, the Pareto optimal front will reach a steady structure, similar to the limit provided by a continuous network definition. More extensive simulations are needed to verify this assumption.

Impact of the flow geometry. The impact of the geometry of a source destination flow on the Pareto optimal set is analyzed in the following. Three flows with different source-destination distances and orientations are defined

Table 1

Variables and parameters of the cross-layer model.

\begin{tabular}{lll}
\hline Node probability & $\chi_{i}$ & Probability node $i$ rebroadcasts a received packet as a function of $x_{i}$ and $\xi_{i}$ \\
Forwarding probability & $x_{i}$ & Probability node $i$ decides to retransmit a packet \\
External impact factor & $\xi_{i}$ & Factor to account for any effect not originating from routing \\
Link probability & $p_{i j}(r)$ & Probability of successful transmission over link $(i, j)$ in resource $r$ \\
Global link probability & $p_{i j}$ & Probability of successful transmission over link $(i, j)$ \\
Transmission rate & $\tau_{i}(r)$ & Percentage of time node $i$ transmits using resource $r$ \\
\hline
\end{tabular}




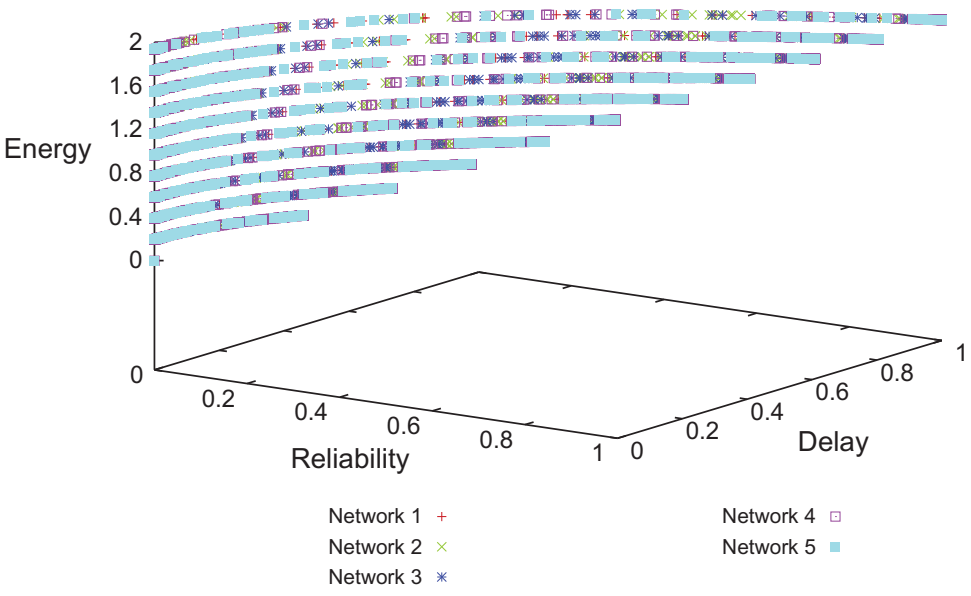

Fig. 11. 1-Relay Pareto optimal sets for five network instances with $\rho=0.004$ for the same $S-D$ flow.

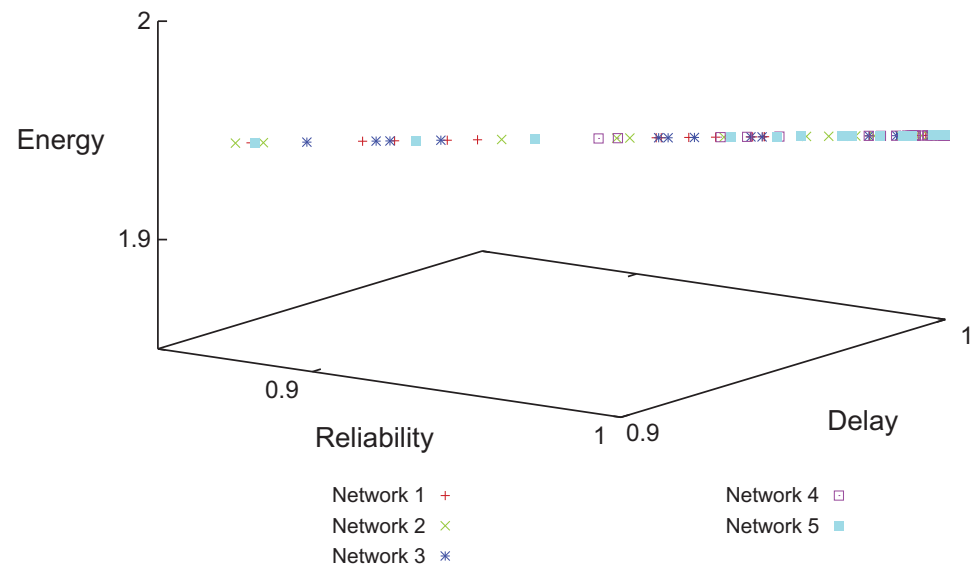

Fig. 12. 1-Relay Pareto optimal sets for five network instances with $\rho=0.004$ for the same $S-D$ flow for highly reliable solutions (zoom in).

as shown in Fig. 14. The 1-relay problem has been solved for each one of the flows separately and their corresponding Pareto optimal sets are given in Fig. 15. A 3-D view and the projection on the reliability-delay plane is plotted.

The orientation of the flows does not impact the tradeoffs between reliability, delay and energy because the distribution of the relays is uniform. Hence, it is the distance between the source and the destination that determines the geometry of the Pareto sets. On the reliability-delay projection, the point where the curve intersects the reliability axis gives the point where delay is null, which means that no relay participates in the transmission. The reliability achieved in this case is a function of the source-destination distance. The shorter this distance gets, the higher the reliability of the no-relay solution gets as well. For the case where source and destination nodes are close enough to have a perfect direct transmission, the Pareto optimal sets is reduced to this unique no-relay solution. Except for this particular case, the sets follow the same trade-offs between reliability, delay and energy.

On the same projection, the point where reliability equals 1 is obtained by several solutions. It can be seen that as the source and the destination get closer, the corresponding transmission delay lowers for a reliability of 1 . Since the direct transmission between $S$ and $D$ has a better link probability, the relay does not need to transmit packets as often to guarantee the reception of the data. Hence, its activity is reduced, and consequently the transmission delay is reduced as well. This of course similarly impacts the energy consumption of the network.

For higher order $M$ - relay problems with $M>1$, the same conclusions can be drawn regarding the impact of the geometry of the flows since the distribution of the nodes is uniform. To go further, it would be interesting to see what kind of Pareto optimal sets are obtained for other types of networks and how flows or other network parameters influence the performance of these networks.

\subsection{Performance comparison with existing routing protocols}

In this section, the performance of Dynamic Source Routing (DSR) [42] and Ad hoc On-Demand Distance Vector (AODV) [43] routing protocols are compared to the Pareto-optimal performance bounds derived with our 


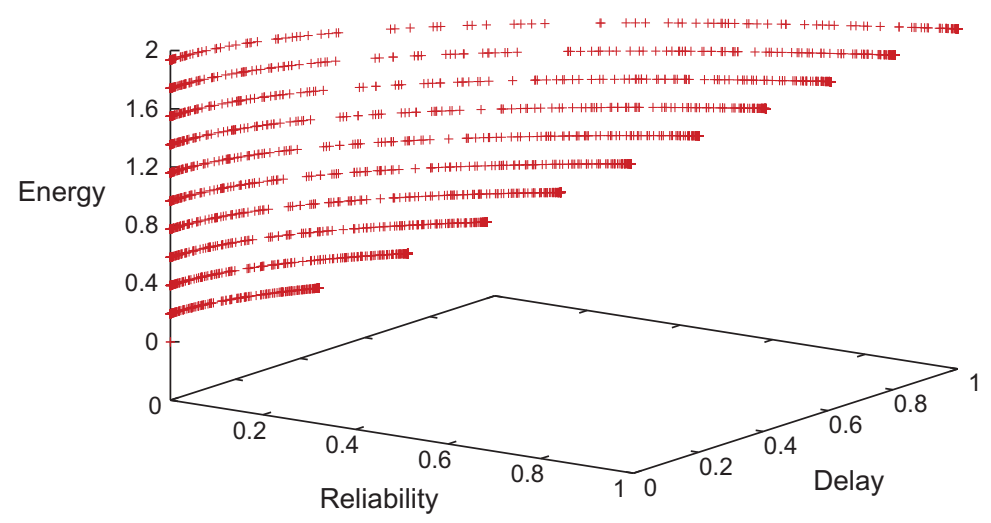

(a)

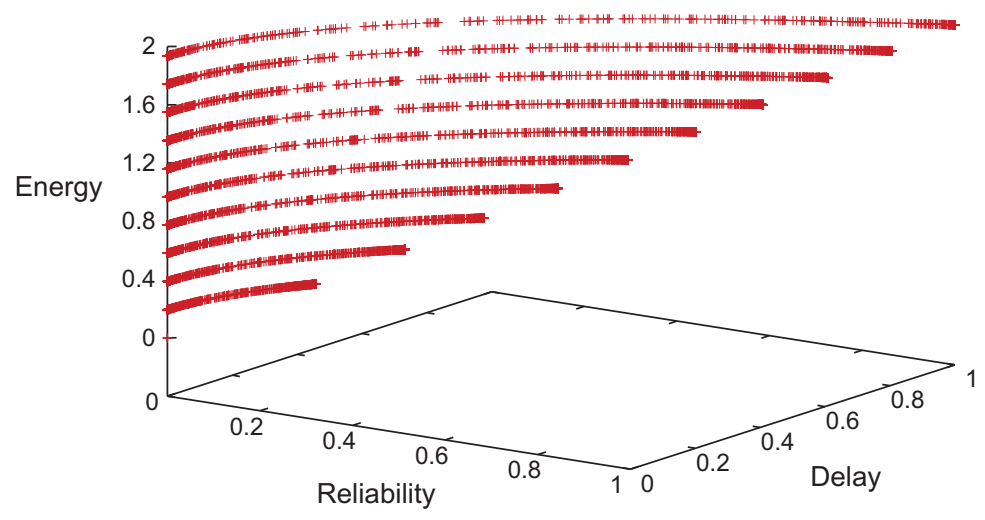

(b)

Fig. 13. Impact of the network density on the 1-relay Pareto optimal set. Density in (a) (resp. (b)) is twice (resp. six times) the density $\rho_{0}=0.004$. (a) has a set of 4176 solutions and (b) has a set of 12,200 solutions.

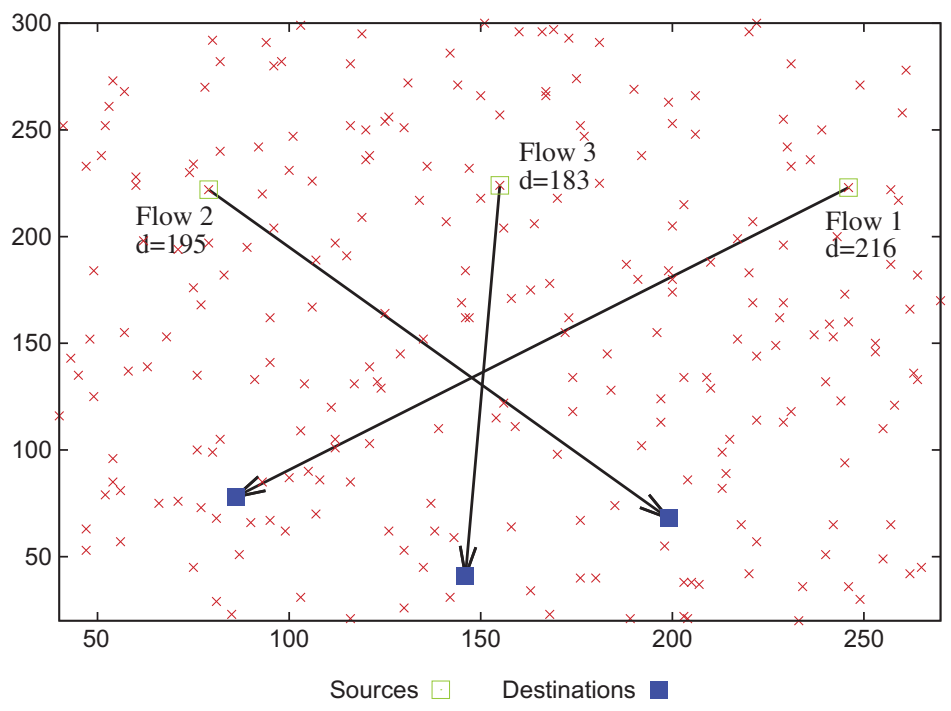

Fig. 14. The three considered flows with their respective source-destination distance.

framework. Our goal is to show how close these two wellknown routing strategies perform to the Pareto optimal choices derived earlier.
6.6.1. DSR and AODV routing protocols

DSR and AODV are reactive routing protocols, where the routes are established on-demand by the source node. To 

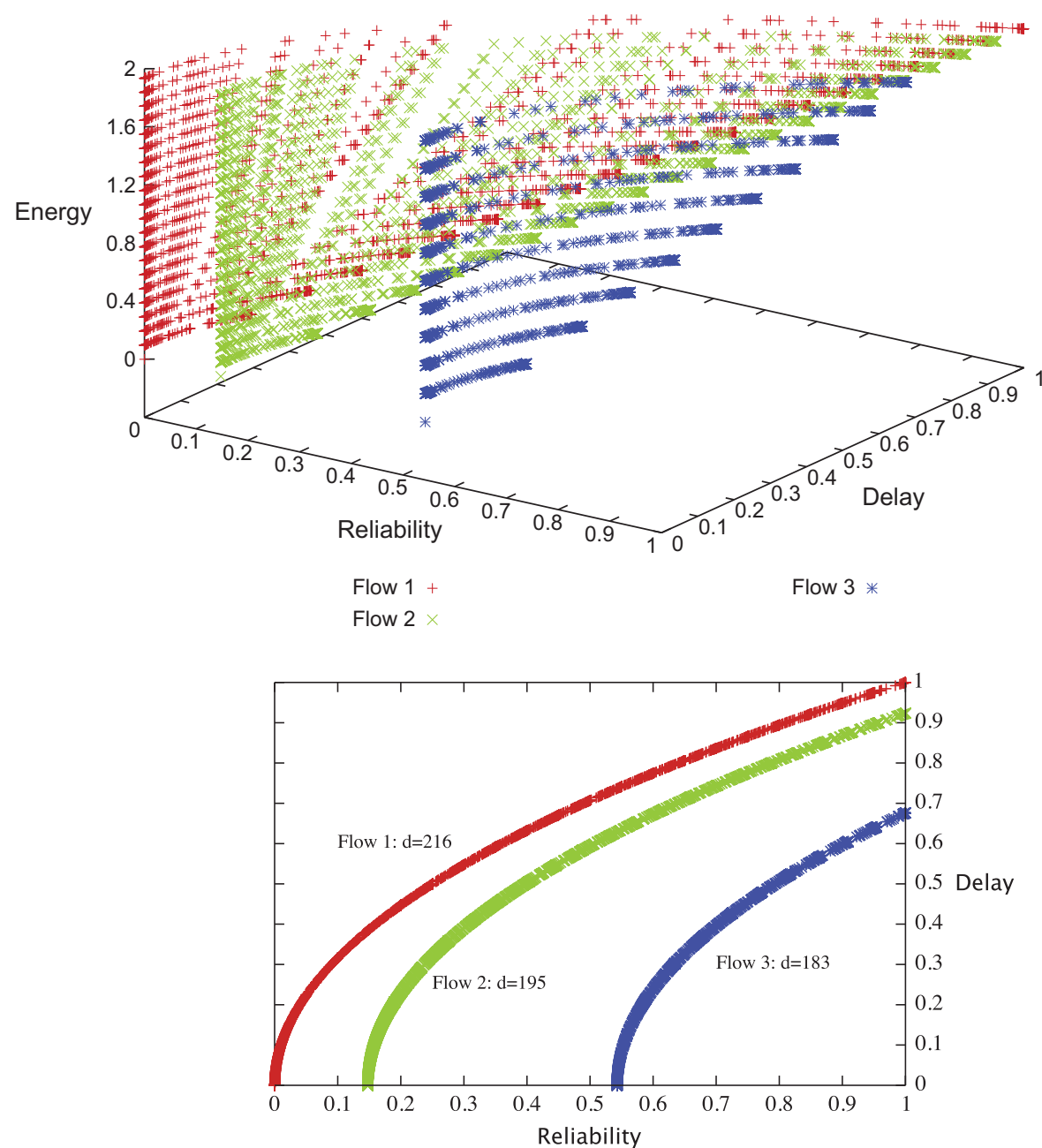

Fig. 15. Reliability delay projection for the three flows.

establish a route, a Route Request (RREQ) packet is broadcast to all nodes of the network by the source. Once the intermediate nodes receive the RREQ packet for the first time, they will broadcast it again to their neighbors if they do not know any route to the destination. A Route Reply packet (RREP) is transmitted to the source node either by the destination or any relay receiving a RREQ packet and knowing a route to the destination. In these algorithms, the destination sends a RREP after it receives the first RREQ. Thus, they generally select the shortest path in cumulative hop count metric. With AODV, each node stores the next-hop neighbor address for each source destination path, while in DSR, a route cache memory stores the completes routes to destination nodes.

With DSR, the header of the RREQ contains the route it has travelled up to arriving at the destination. In this case, the packet header can be used to store cumulative metric values (e.g. hop count, cumulative geometrical distance, cumulative packet error rate, ...). The destination can then wait for several RREQ packets to arrive from multiple routes and select the one with the best metric value (minimum geometrical distance, PER..) to improve reliability for instance [44]. Multiple routes per destination may be maintained [45]. In this section, we target a DSR implementation where only one route is selected and maintained. Important to both algorithms, the neighborhood of each relay has to be maintained using HELLO messages to make sure the next hop node is still in range.

In this section, two DSR routing implementations are simulated to derive the routing path relative to two different metrics: the shortest cumulative distance path from $S$ to $D$ and the path with the minimum cumulative PER. For each implementation, two nodes are defined as neighbors in the protocol if the average link PER is smaller or equal to $\Delta \geqslant 0$.

For AODV, the shortest hop-count path is selected. It resumes to choosing, at each hop, the relay which is the closest to the destination among the set of neighbor nodes which are reachable with perfectly reliable links (i.e. with PER $=0$ ).

\subsubsection{Simulation setup}

To be consistent with the simulation of the previously defined 1- and 2-relay problems, we consider a frame 
Table 3

Analytical and simulated performance of DSR and AODV routing protocols for different neighborhood selection strategies ( $\Delta$ values).

\begin{tabular}{|c|c|c|c|c|c|}
\hline \multirow[t]{2}{*}{ Routing protocol } & \multirow[t]{2}{*}{$\Delta$} & \multirow[t]{2}{*}{ Routing path } & \multicolumn{2}{|c|}{ Performance evaluation } & \multirow[t]{2}{*}{ Neighborhood size } \\
\hline & & & Analytical & By simulation & \\
\hline DSR-dist solution 1 & $\Delta>0$ & $\mathrm{~S} \rightarrow$ Node $56 \rightarrow \mathrm{D}$ & $\begin{array}{l}f_{R}=1-10^{-8} \\
f_{D}=1 \\
f_{E}=1.931\end{array}$ & $\begin{array}{l}f_{R}=1 \\
f_{D}=1 \\
f_{E}=1.934\end{array}$ & $\begin{array}{l}\text { S: } 196 \\
\text { Node } 56: 333(\Delta=0.1) \\
\text { S: } 177 \\
\text { Node } 56: 296(\Delta=0.01) \\
\text { S:164 } \\
\text { Node } 56: 261(\Delta=0.001)\end{array}$ \\
\hline DSR-dist solution 2 & 0 & $\mathrm{~S} \rightarrow$ Node $56 \rightarrow$ Node $58 \rightarrow \mathrm{D}$ & $\begin{array}{l}f_{R}=1 \\
f_{D}=2 \\
f_{E}=3.862\end{array}$ & $\begin{array}{l}f_{R}=1 \\
f_{D}=2 \\
f_{E}=3.868\end{array}$ & $\begin{array}{l}\text { S: } 102 \\
\text { Node } 56: 133 \\
\text { Node } 58: 133\end{array}$ \\
\hline DSR-PER solution 3 & $\Delta>0$ & $\mathrm{~S} \rightarrow$ Node $56 \rightarrow \mathrm{D}$ & $\begin{array}{l}f_{R}=1-10^{-8} \\
f_{D}=1 \\
f_{E}=1.931\end{array}$ & $\begin{array}{l}f_{R}=1 \\
f_{D}=1 \\
f_{E}=1.934\end{array}$ & $\begin{array}{l}\text { S: } 196 \\
\text { Node 56: } 333(\Delta=0.1) \\
\text { S: } 177 \\
\text { Node 56: } 296(\Delta=0.01) \\
\text { S:164 } \\
\text { Node 56: } 261(\Delta=0.001)\end{array}$ \\
\hline DSR-PER solution 4 & 0 & $\mathrm{~S} \rightarrow$ Node $23 \rightarrow$ Node $4 \rightarrow \mathrm{D}$ & $\begin{array}{l}f_{R}=1 \\
f_{D}=2 \\
f_{E}=3.862\end{array}$ & $\begin{array}{l}f_{R}=1 \\
f_{D}=2 \\
f_{E}=3.868\end{array}$ & $\begin{array}{l}\text { S: } 102 \\
\text { Node 23: } 121 \\
\text { Node 4: } 119\end{array}$ \\
\hline AODV solution & 0 & $\mathrm{~S} \rightarrow$ Node $56 \rightarrow$ Node $92 \rightarrow \mathrm{D}$ & $\begin{array}{l}f_{R}=1 \\
f_{D}=2 \\
f_{E}=3.862\end{array}$ & $\begin{array}{l}f_{R}=1 \\
f_{D}=2 \\
f_{E}=3.868\end{array}$ & $\begin{array}{l}\text { S: } 102 \\
\text { Node 56: } 133 \\
\text { Node 92: } 116\end{array}$ \\
\hline
\end{tabular}

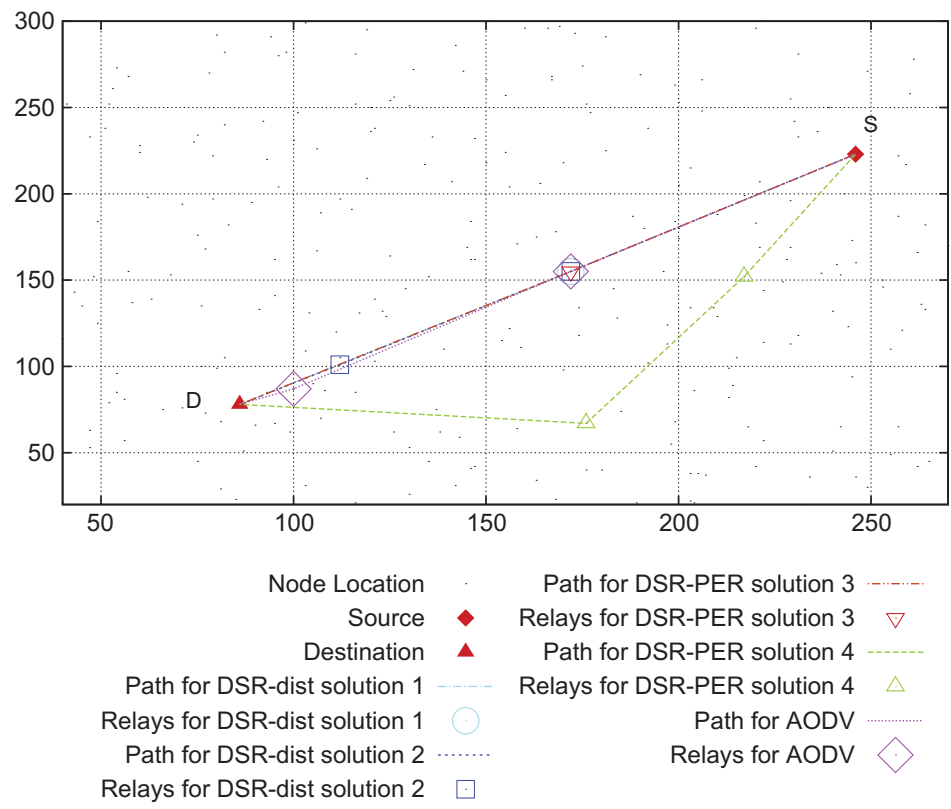

Fig. 16. Routing solutions for DSR and AODV routing protocols.

composed of $R=2$ time slots. The same network topology as defined in Section 6.1 is considered. The source is transmitting a packet in the first time slot while the relays share the second time slot for relaying. Since several relays may participate in the relaying, we adopt the IEEE 802.11 DCF MAC protocol to grant access to the relays in the second slot. The overhead due to routing and MAC is not measured in our simulation in order to provide a fair comparison with our framework results. Indeed, the framework does not account for any protocol overhead in its definition.

In this study, three routing implementations are tested: DSR with minimum cumulative distance (DSR-dist), DSR with minimum cumulative PER (DSR-PER) and AODV which minimizes the hop-count metric. For the two DSR implementations, neighborhood sets are adjusted based on the value of $\Delta$, with $\Delta \in\left\{0,10^{-3}, 10^{-2}, 10^{-1}\right\}$. Smaller values of $\Delta$ select neighbor nodes with highly reliable links, inducing neighborhood sets of smaller cardinality. For the DSR-PER implementation, which looks for the maximum reliability route, a possibly very big number of routes with a zero cumulative PER may exist because of multi-hop forwarding. Thus, since in our paper we have investigated 1 and 2-relay problems, we will look for the route with maximum reliability composed of at most 1 

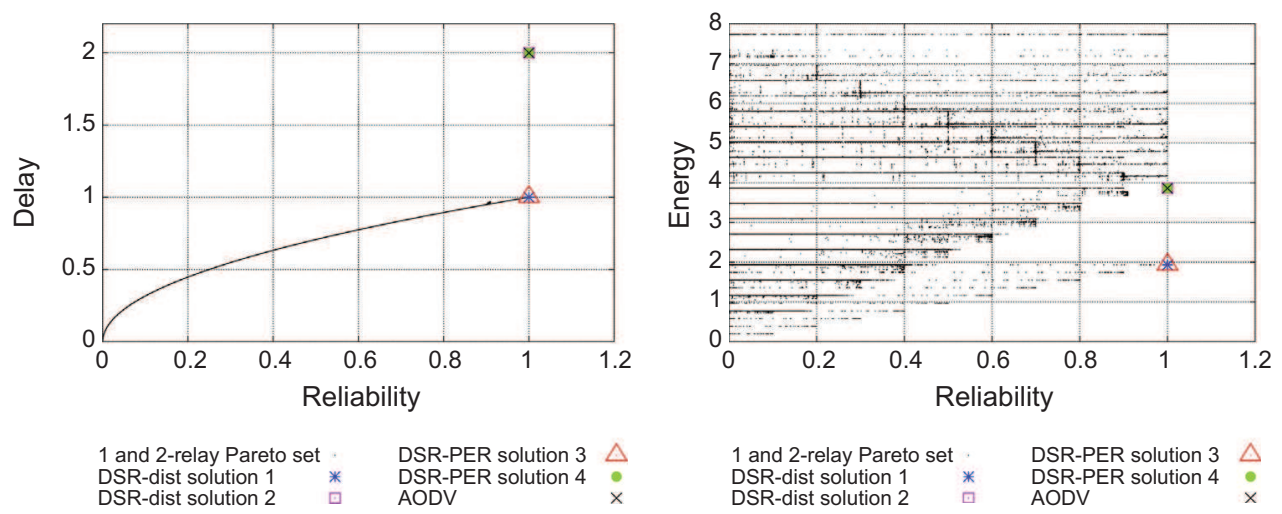

$\begin{array}{llll}1 \text { and 2-relay Pareto set } & & \text { DSR-PER solution 3 } & \triangle \\ \text { DSR-dist solution 1 } & * & \text { DSR-PER solution 4 } & \bullet \\ \text { DSR-dist solution 2 } & & \text { AODV } & \times\end{array}$

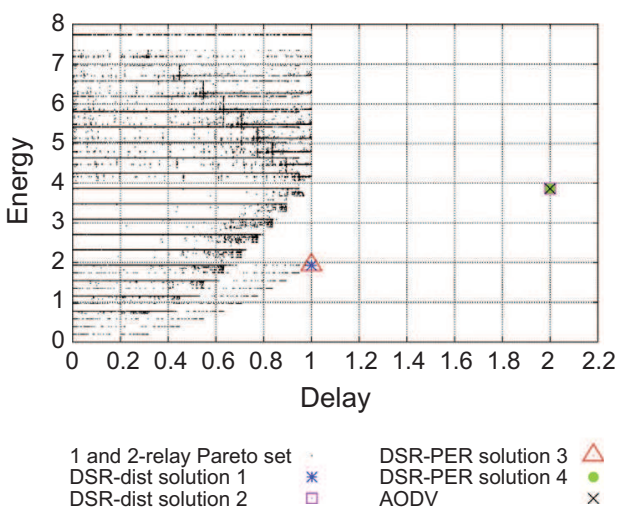

Fig. 17. The Pareto optimal set and DSR and AODV routing results.

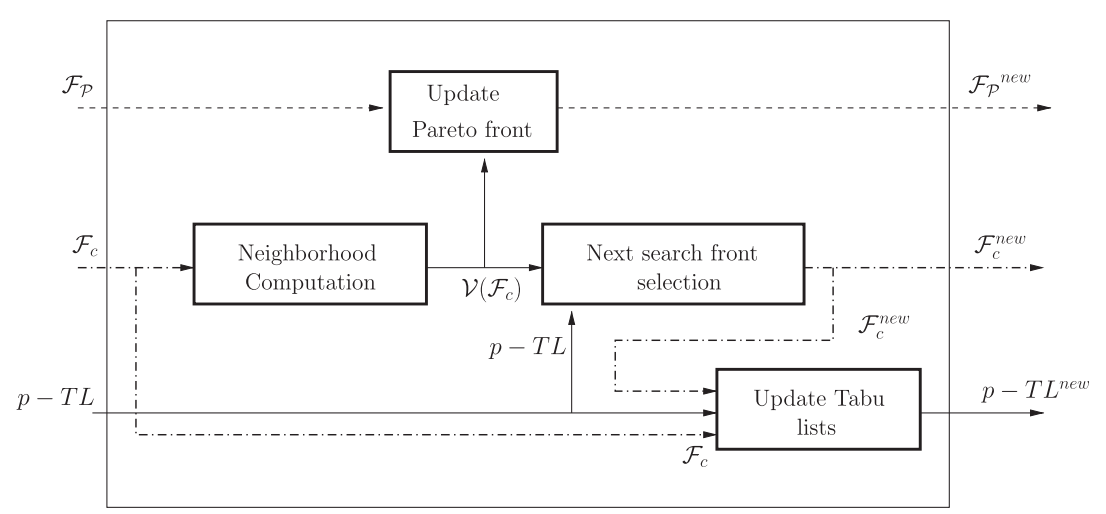

Fig. A.18. PMOTS: Description of one search iteration.

or 2 relays. It is equivalent to introducing a Time To Live (TTL) field in the RREQ packet that specifies a maximum number of 2 or 3 hops.

In the simulations, routes are first calculated for the appropriate routing metric using Dijkstra's algorithm either for a reliable neighborhood sets $(\Delta=0)$ or for unreliable neighborhood sets $\left(\Delta \in\left\{10^{-3}, 10^{-2}, 10^{-1}\right\}\right)$. Then, a flow of 10,000 packets is transmitted on the selected route. The performance evaluation criteria of the resulting routing solution is calculated analytically and by simulation as stated earlier in Sections 4 and 6.4 , respectively.

\subsubsection{Results}

The analytical and simulated results for the three routing protocols are shown in Table 3. The routing path calculated for each routing implementation is given, together with the analytical and simulated values of $f_{R}, f_{D}$ and $f_{E}$. The impact of $\Delta$ on the neighborhood is represented as well in Table 3 by showing for the source and relay nodes the size of their neighborhood. Fig. 16 represents the location of the different routes in the network and Fig. 17 compares the evaluations in $f_{R}, f_{D}$ and $f_{E}$ of each routing solution to our Pareto-optimal performance bound for the 1-relay and 2-relay problem. 

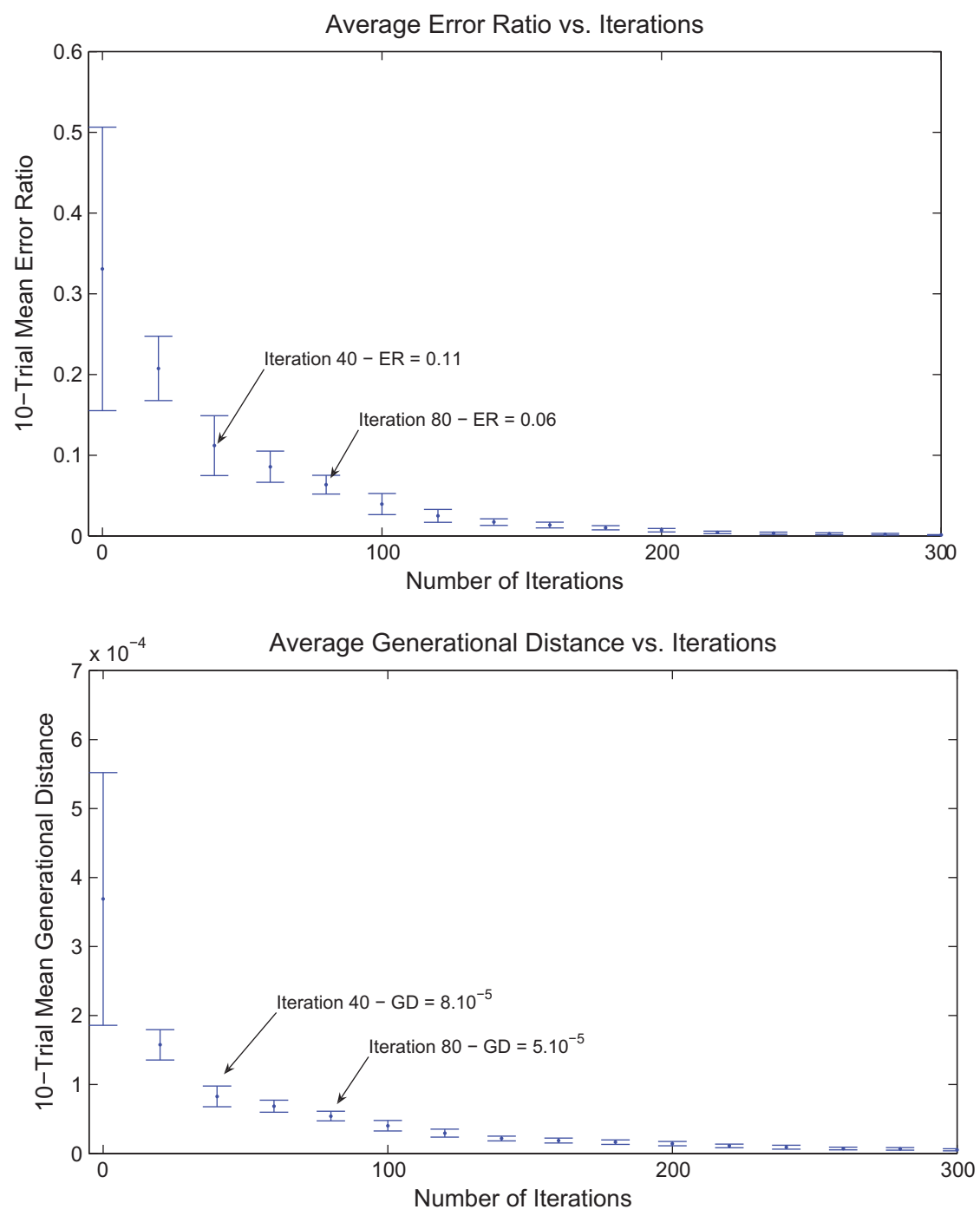

Fig. B.19. PMOTS performance: error ratio and generational distance statistics for the 1-relay problem.

For the tested implementations, different routes are obtained:

- For DSR-dist, two routing solutions are obtained, referred to as DSR-dist solutions 1 and 2. DSR solution 1 is obtained as unreliable neighbors are permitted (i.e. $\Delta>0$ ) and is thus composed of a single relay. DSR solution 2 is constructed as only perfectly reliable relays are selected as neighbors, reducing the neighborhood set size. Therefore, the DSR-dist solution 2 is composed of 2 relays, adding another relay to DSR-dist solution 1 on the source-destination path.

- For DSR-PER, two solutions referred to as DSR-PER solutions 3 and 4 are obtained. Solution 3 is obtained when unreliable neighbors are permitted and a TTL of 2 hops is enforced while solution 4 is obtained when $\Delta=0$ and a TTL of 3 hops is chosen. DSR-PER solution 3 is exactly the same solution as DSR-dist solution because the propagation model we use is a direct function of the distance. DSR-PER solution 4 is one of the solutions with a zero cumulative PER and a TTL of 3 hops.

- For AODV, the shortest hop-count route contains two relays for this topology. The second relay of the AODV solution is different from the one of DSR-dist solution 2. This is due to the fact that AODV selects the second relay that is the closest to the destination, and not the one that minimizes the cumulative path distance.

Over all tested scenarios, analytical and simulated values of the optimization criteria are really close, showing that the analytical calculation provides (for this topology) a good performance measure even if CSMA/CA protocol is introduced in the second time slot. All routing solutions provide a close to one reliability criterion. DSR-PER solution 3 (which is equal to DSR-dist solution 1) maximizes the reliability of the 1-relay case. As shown in Fig. 17, DSR-PER corresponds exactly to the solution of 

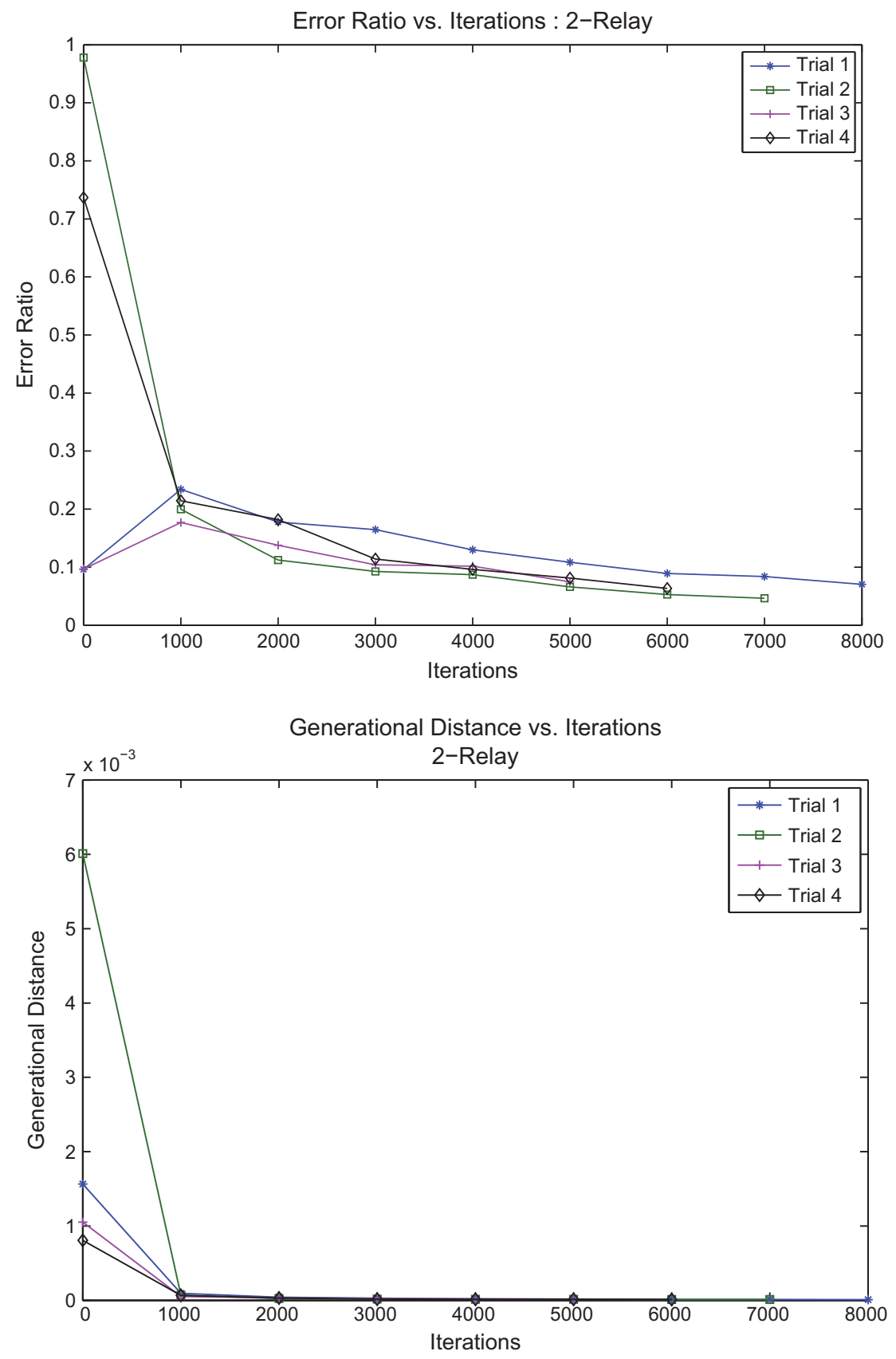

Fig. B.20. PMOTS performance: error ratio and generational distance for the 2-relay problem.

the 1-relay problem with maximum reliability. For the other solutions different delay and energy criteria are obtained. This is due to the fact that conventional multihop routing does not leverage multi-path as we do in the framework. Routes with 3 hops have a delay criterion of 2 because all packets travel on a 2-hop path, whether the retransmission of the first relay can reach the destination or not. With our framework, the packets transmitted by the 2-relay solutions travel in average on a 2-hop path (and not a 3-hop one) and therefore have an average delay of 1 . Since most of the time the transmission through one of the relays located in between $S$ and $D$ (cf. Fig. 9) is successful, the second transmission is not necessary.

We can conclude that the three routing implementations leading to DSR-dist solution 2, DSR-PER solution 4 and AODV are dominated by the Pareto-optimal solutions of our multiobjective performance bound. Routing strategies leading to DSR-dist solution 1 and DSR-PER solution 3 , where unreliable neighbor links are leveraged, are Pareto-optimal in the sense of maximum reliability, minimum average delay and minimum average energy consumption.

More generally, we have shown that it is topical to compare the performance of state-of-the-art routing solutions 
to our Pareto-optimal performance bounds. Several simple routing implementations have been tested, showing that some of them are Pareto-optimal while others are dominated by the derived bound.

\section{Conclusion}

In this paper, we have proposed a novel multiobjective optimization framework for network routing and resource allocation in wireless ad hoc networks. Our proposed framework consists of a general probabilistic network model capable of capturing the impact and interaction of a wide range of resource/interference management techniques, various channel conditions, and network scenarios. Used in conjunction with metaheuristic optimization techniques, this framework provides an efficient tool to capture the trade-offs between different performance metrics and to obtain bounds on the achievable performance of routing for a single source-destination transmission. Results were obtained for characterizing the delay, reliability, and energy trade-offs for a two time slot sensor network model. Future work will extend the model to consider more complex network scenarios, for example accounting for various network topologies, including multiple concurrent flows in the network, and using more refined cross-layer interaction and interference models. Other development will introduce a continuous network model in order to get asymptotic Pareto optimal sets for large scale networks. In the same sense, we will target as well networks of mobile nodes by leveraging statistical mobility models of the literature. In this case, links between nodes are a function of their probability to meet with a sufficient channel capacity.

\section{Acknowledgments}

This work was supported in part by the Marie Curie IOF Action of the European Community's Sixth Framework Program (DistMO4WNet \#MOIF-CT-2006-040724 by the ONR Grant \#N00014-06-1-0063, and by the NSF AGEP Grant \#HRD-0450360. This article only reflects the authors' views and neither European Community, ONR, nor AGEP is liable for any use that may be made of the information contained herein. Preliminary results for this work were presented to IEEE WiOpt 2010 in Avignon, France.

The authors thank Dr. Wayne Phoel, Dr. Jeffrey Wysocarski, and Dr. Michele Schuman of MIT Lincoln Laboratory for their useful discussions and insightful feedback.

\section{Appendix A. PMOTS algorithm}

This appendix presents briefly a multiobjective metaheuristic called PMOTS (Parallel MultiObjective Tabu Search) that has been presented in [9]. It is based on the well known Tabu metaheuristic [36]. PMOTS is a multiobjective extension of Tabu search where $K$ Tabu searches are performed in parallel. Its macro-algorithm is given in Algorithm 2 and a graphical description is shown in Fig. A.18. The goal of this algorithm is to obtain the best possible approximation of the Pareto optimal set of solutions $F_{P}$.

In a search iteration, $i$, the $K$ parallel search paths are represented as a search set or search front $F_{c}(i)$ of $K$ solutions. The first set of $K$ solutions is randomly created. A set of neighbor solutions $V\left(S_{k}\right)$ for each solution $S_{k}$ of the search front is computed according to a set of rules. The neighborhood generation rules specific to our MO problem enable atomic changes to solution $S_{k}$ by altering the selection of relay nodes, the transmission rates $\tau_{i}$, and/or the resources $r$. Further details on these rules can be found in [9]. The pool of neighbor solutions is added to the current Pareto optimal front $F_{P}$, and the new front $F_{P}^{\text {new }}$ is obtained by extracting the set of non-dominated solutions.

A new search front $F_{c}(i+1)$ is selected by choosing promising non-Tabu solutions that are not always nondominated to avoid a premature convergence of the algorithm. Therefore for a path $k$, each new solution is selected randomly in the set of neighbor solutions of $S_{k}$ which is limited to the solutions having a Pareto rank $R_{\max }$. The rank of a solution $x$ is defined by $R(x)=1+d(x)$, where $d(x)$ is the number of solutions by which $x$ is dominated in the set of feasible solutions $S$. The solutions of the Pareto optimal set have a $\operatorname{rank} R(x)=1$. In this algorithm, the Pareto ranking is local to the set of neighbor solutions and does not include the current estimated Pareto set $F_{P}$. By not including $F_{P}$ and selecting fairly good solutions with the Pareto rank constraint, diversity is introduced within the search strategy. Once $F_{c}(i+1)$ is chosen, the solutions of $F_{c}(i)$ are stored in the corresponding Tabu lists.

There is also a restart strategy that creates a new random search front if no solutions have been added to or suppressed from $F_{P}$ for a given number of search iterations. The algorithm stops after a fixed number of iterations and provides an estimate of the Pareto front $F_{P}$.

\section{Algorithm 2. Macro-Algorithm for PMOTS.}

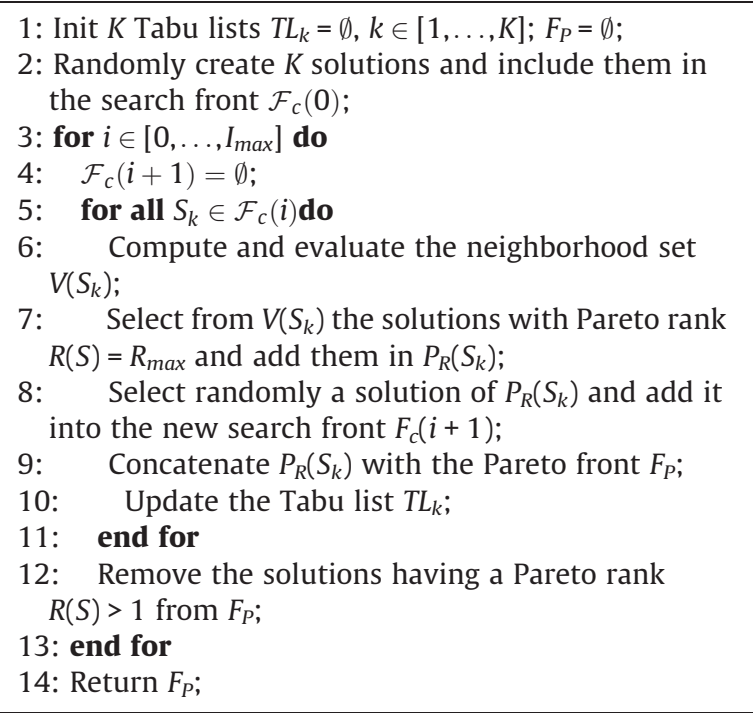

\section{Appendix B. Validation of PMOTS}

The proposed multiobjective probabilistic network model provides a coherent and complete view of the 
trade-offs that arise between reliability, delay, and energy. For larger networks or when more relays are needed, our problem is solved using the multiobjective optimization algorithm PMOTS as presented in Section 4.2. This appendix presents results related to the convergence properties of PMOTS for the 1-relay and 2-relay problems.

Two performance metrics measure the convergence of PMOTS towards the Pareto optimal set $F_{p}^{*}$ obtained through exhaustive search. The approximated Pareto sets $F_{P}$ obtained by PMOTS are compared to $F_{P}^{*}$ with respect to the number of iterations the search has performed using the following metrics [46]:

- The error ratio that measures the non-convergence of a search method to $F_{P}^{*}$. It is given by:

$$
E R=\frac{\sum_{i=1}^{n} e_{i}}{n}
$$

where $e_{i}=0$ if solution $i$ of $F_{P}$ belongs to $F_{P}^{*}$ and $e_{i}=1$ otherwise, and $n$ is the number of solutions in the approximated Pareto front $F_{P}$.

- The generational distance that measures the distance between an estimated set of $n$ solutions and the theoretical Pareto front $F_{P}^{*}$. It is defined by:

$$
G D=\frac{\left(\sum_{i=1}^{n} d_{i}^{p}\right)^{1 / p}}{n}
$$

where $d_{i}$ is the smallest distance between a solution of $F_{P}$ and $F_{p}^{*}$. Here, we use $p=2$ and $n$ the number of solutions of the approximated Pareto front $F_{P}$.

The smaller the error ratio and the generational distance metrics, the smaller in number and amplitude are the errors between $F_{P}$ and $F_{p}^{*}$. These metrics have been calculated for the 1-relay Pareto fronts obtained with PMOTS every 20 iterations. Average and standard deviation values are computed over 10 runs of PMOTS using the same test environment. In Fig. B.19, it can be seen that both ER and $G D$ quickly decrease with time. Iterations 40 and 80 have been highlighted in Fig. B.19 because they represent the times at which PMOTS has evaluated the number of solutions equal to half the search space and the entire search space, respectively. At iteration 80 , only about $4 \%$ of the solutions of $F_{P}$ do not belong to $F_{p}^{*}$, and these solutions are really close to the Pareto optimal front as shown by the $G D$ measure of $7 \times 10^{-5}$. At iteration 40 , we already have a good first picture of the Pareto optimal front. Indeed, the generational distance shows that the erroneous solutions of $F_{P}$ are very close to $F_{P}^{*}$ with a value of $G D=1 \times 10^{-4}$. Moreover, looking at the percentage of solutions of $F_{P}$ that belong to $F_{P}^{*}$, we hare already identified 60\% of the Pareto-optimal solutions.

Similar analysis has been pursued with the 2-relay Pareto optimal set. Fig. B.20 shows the performance of four runs of the PMOTS algorithm for the 2-relay problem using the same test environment. The error ratio and generational distance values are calculated after the initial neighborhood generation and at every 1000 iterations of the algorithm. In two of the cases, the initial solution selected for neighborhood exploration provides a very poor initial estimate. By 1000 iterations of PMOTS, however, we obtain a good estimate of the Pareto front. In all four runs, the error ratio is below 25\% at iteration 1000 . The maximum generational distance for the trials at iteration 1000 is $G D=9.37^{-5}$. Although there are still more non-dominated solutions to obtain in further iterations, we have confidence in the initial observations of the performance trade-offs. PMOTS performs well for the 1-relay and 2-relay investigation. As we increase to even larger problem instances, we will continue to improve the algorithm's implementation and convergence speed.

\section{References}

[1] M. Brand, P. Maymounkov, A.F. Molisch, Routing with probabilistic delay guarantees in wireless ad hoc networks, in: IEEE GLOBECOM, New Orleans, LA, USA, November 2008, pp. 1-6.

[2] N. Vassileva, F. Barcelo-Arroyo, A survey of routing protocols for energy constrained ad hoc wireless networks, in: Proceedings of the Future Generation Communication and Networking (FGCN), Korea, vol. 1,December, 2007, pp. 522-527.

[3] A. Eryilmaz, R. Srikant, Joint congestion control, routing, and MAC for stability and fairness in wireless networks, IEEE Journal on Selected Areas in Communications 24 (2006) 1514-1524.

[4] C. Touati, E. Altman, J. Galtier, Fair power and transmission rate control in wireless networks, in: IEEE GLOBECOM, Taipei, Taiwan, R.O.C, vol. 2, November 2002, pp. 1229-1233.

[5] L. Shi, A.O. Fapojuwo, Cross-layer optimization with cooperative communication for minimum power cost in packet error rate constrained wireless sensor networks, Ad Hoc Networks 10 (2012) 1457-1468.

[6] A. Goldsmith, M. Effros, R. Koetter, M. Mèdard, A. Ozdaglar, L. Zheng, Beyond Shannon: the quest for fundamental performance limits of wireless ad hoc networks, IEEE Communications Magazine 49 (2011) 195-205.

[7] B.C. Villaverde, S. Rea, D. Pesch, Multi-objective cross-layer algorithm for routing over wireless sensor networks, in: Third International Conference on Sensor Technologies and Applications (SENSORCOMM), Athens/Glyfada, Greece, June 2009, pp. 568-574.

[8] S. He, J. Chen, W. Xu, Y. Sun, P. Thulasiraman, X.S. Shen, A stochastic multiobjective optimization framework for wireless sensor networks, EURASIP Journal on Wireless Communications and Networking 2010 (2010) 1-11.

[9] K. Jaffrès-Runser, J.-M. Gorce, C. Comaniciu, A multiobjective Tabu framework for the optimization and evaluation of wireless systems, in: W. Jaziri (Ed.), Local Search Techniques: Focus on Tabu Search, ITech Education and Publishing, 2008, pp. 29-54.

[10] K. Jaffrès-Runser, M.R. Schurgot, C. Comaniciu, J.-M. Gorce, A multiobjective performance evaluation framework for routing in wireless ad hoc networks, in: Proceedings of the 8th International Symposium on Modeling and Optimization in Mobile, Ad Hoc and Wireless Networks (WiOpt), Avignon, France, May 2010, pp. 113-121.

[11] V.P. Mhatre, C.P. Rosenberg, R.R. Mazumdar, On the capacity of ad hoc networks under random packet losses, IEEE Transactions on Information Theory 55 (2009) 2494-2498.

[12] Z. Wang, H.R. Sadjadpour, J.J. Garcia-Luna-Aceves, A unifying perspective on the capacity of wireless ad hoc networks, in: Proceedings of IEEE INFOCOM, Phoenix, USA, April 2008, pp. 211215.

[13] A.F. Dana, R. Gowaikar, R. Palanki, B. Hassibi, M. Effros, Capacity of wireless erasure networks, IEEE Transactions on Information Theory 52 (2006) 789-804.

[14] P. Gupta, P.R. Kumar, The capacity of wireless networks, IEEE Transactions on Information Theory 46 (2000) 388-404.

[15] M. Grossglauser, D. Tse, Mobility increases the capacity of ad hoc wireless networks, IEEE/ACM Transactions on Networking 10 (2002) 477-486.

[16] Z. Wang, H. Sadjadpour, J. Garcia-Luna-Aceves, S. Karande, Fundamental limits of information dissemination in wireless ad hoc networks - Part I: Single-packet reception, IEEE Transactions on Wireless Communications 8 (2009) 5749-5754.

[17] Z. Wang, H. Sadjadpour, J. Garcia-Luna-Aceves, Fundamental limits of information dissemination in wireless ad hoc networks - Part II: Multi-packet reception, IEEE Transactions on Wireless Communications 10 (2011) 803-813. 
[18] A. El Gamal, J. Mammen, B. Prabhakar, D. Shah, Throughput-delay trade-off in wireless networks, in: Proceedings of IEEE INFOCOM, Hong Kong, China, vol. 1, March 2004, pp. 464-475.

[19] C. Comaniciu, H.V. Poor, On the capacity of mobile ad hoc networks with delay constraints, IEEE Transactions on Wireless Communications 5 (2006) 2061-2071.

[20] C. Bae, W.E. Stark, Energy-bandwidth tradeoff with spatial reuse in wireless multi-hop networks, in: Military Communications Conference (MILCOM), San Diego, CA, November 2008, pp. 1-7.

[21] M. Tahir, R. Farrell, A cross-layer framework for optimal delaymargin, network lifetime and utility tradeoff in wireless visual sensor networks, Ad Hoc Networks 11 (2013) 701-711.

[22] M. Brand, A.F. Molisch, Delay-energy tradeoffs in wireless ad-hoc networks with partial channel state information, in: IEEE GLOBECOM, New Orleans, LA, USA, November 2008, pp. 1-6.

[23] F. Xue, A. Sanderson, R. Graves, Multi-objective routing in wireless sensor networks with a differential evolution algorithm, in: Proceedings of the IEEE International Conference on Networking, Sensing and Control (ICNSC), Florida, USA, April 2006, pp. 880-885.

[24] R. Zhang, O. Berder, J.-M. Gorce, O. Sentieys, Energy delay tradeoff in wireless multihop networks with unreliable links, Ad Hoc Networks 10 (2012) 1306-1321.

[25] J.-M. Gorce, R. Zhang, K. Jaffrès-Runser, C. Goursaud, Energy, latency and capacity trade-offs in wireless multi-hop networks, in: IEEE 21 st International Symposium on Personal, Indoor and Mobile Radio Communications (PIMRC), Istanbul, Turkey, September 2010, pp. 2755-2760.

[26] S. Toumpis, A. Goldsmith, Performance, optimization, and crosslayer design of media access protocols for wireless ad hoc networks, in: IEEE International Conference on Communications (ICC), Anchorage, Alaska, USA, vol. 3, May 2003, pp. 2234-2240.

[27] J. Luo, C. Rosenberg, A. Girard, Engineering wireless mesh networks: joint scheduling, routing, power control, and rate adaptation, IEEE/ ACM Transactions on Networking 18 (2010) 1387-1400.

[28] J. Luo, A. Iyer, C. Rosenberg, Throughput-lifetime trade-offs in multihop wireless networks under an SINR-based interference model, IEEE Transactions on Mobile Computing 10 (2011) 419-433.

[29] V. Srivastava, M. Motani, Cross-layer design: a survey and the road ahead, IEEE Communications Magazine 43 (2005) 112-119.

[30] G. Song, Y. Li, Cross-layer optimization for OFDM wireless networks - Part I: Theoretical framework, IEEE Transactions on Wireless Communications 4 (2005) 614-624.

[31] U.C. Kozat, I. Koutsopoulos, L. Tassiulas, A framework for cross-layer design of energy-efficient communication with QoS provisioning in multi-hop wireless networks, in: Proceedings of IEEE INFOCOM, Hong Kong, China, vol. 2, March 2004, pp. 1446-1456.

[32] Y. Donoso, R. Fabregat, J.L. Marzo, A multi-objective optimization scheme for multicast routing: a multitree approach, Telecommunication Systems 27 (2004) 229-251.

[33] F. Martins, E. Carrano, E. Wanner, R. Takahashi, G. Mateus, A hybrid multiobjective evolutionary approach for improving the performance of wireless sensor networks, IEEE Sensors Journal 11 (2011) 545-554.

[34] A. Roy, N. Banerjee, S. Das, An efficient multi-objective QoS-routing algorithm for wireless multicasting, in: IEEE 55th Vehicular Technology Conference (VTC), Vancouver, BC, vol. 3, September 2002, pp. 1160-1164.

[35] X. Cui, C. Lin, Y. Wei, A multiobjective model for QoS multicast routing based on genetic algorithm, in: International Conference on Computer Networks and Mobile Computing (ICCNMC), Shanghai, China, October 2003, pp. 49-53.

[36] F. Glover, M. Laguna, Tabu Search, Kluwer Academic Publisher, Boston, 1997.

[37] R. Zhang, J-M. Gorce, Optimal transmission range for minimum energy consumption in wireless sensor networks, in: IEEE Wireless Communications and Networking Conference (WCNC), Las Vegas, March 2008, pp. 757-762.

[38] S. Toumpis, Mother nature knows best: a survey of recent results on wireless networks based on analogies with physics, Computer Networks: The International Journal of Computer and Telecommunications Networking 52 (2008) 360-383.

[39] N. Gast, B. Gaujal, A mean field approach for optimization in discrete time, Discrete Event Dynamic Systems 21 (2011) 63-101.
[40] WSNet, Worldsens Simulator, 2012. <http://wsnet.gforge.inria.fr/>.

[41] P. Jacquet, Geometry of information propagation in massively dense ad hoc networks, in: Proceedings of the 5th ACM International Symposium on Mobile Ad Hoc Networking and Computing (MobiHoc), Tokyo, Japan, May 2004, pp. 157-162.

[42] D.B. Johnson, D.A. Maltz, Dynamic source routing in ad hoc wireless network, in: Mobile Computing, vol. 5, pp. 153-181.

[43] B.E.R.C. Perkins, S. Das, Ad hoc on-demand distance vector (AODV) routing, Internet RFCs, IETF, 2003.

[44] W.-I. Kim, D.-H. Kwon, Y.-J. Suh, A reliable route selection algorithm using global positioning systems in mobile ad-hoc networks, in: IEEE International Conference on Communications, 2001, ICC 2001, vol. 10, pp. 3191-3195.

[45] N.K.R.V. Ramesh, P. Subbaiah, M.J. Raju, Performance comparison and analysis of DSDV and AODV for MANET, International Journal on Computer Science and Engineering (JJCSE) 02 (2010) 183-188.

[46] Y. Collette, P. Siarry, Multiobjective Optimization: Principles and Case Studies, Springer, 2003.

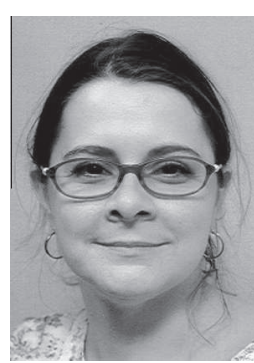

Katia Jaffrès-Runser received her Dipl. Ing. (M.Sc.) in 2002 and her Ph.D. in 2005 from INSA-Lyon, France. From 2002 to 2005 she was with INRIA while working towards her Ph.D. thesis. In 2006, she joined Stevens Institute of Technology, NJ, USA, as a postdoctoral researcher. She is the recipient of a $3-$ year Marie-Curie OIF fellowship to work at both Stevens I.T. and INSA Lyon on multiobjective optimization of wireless networks. In 2011, she was with INRIA, participating in the GreenTouch initiative. Since September 2011, she is an Associate Professor at University of Toulouse, in the Telecommunications and Networking department of INPT-ENSEEIHT. Her main field of interest is the performance evaluation of wireless networks.

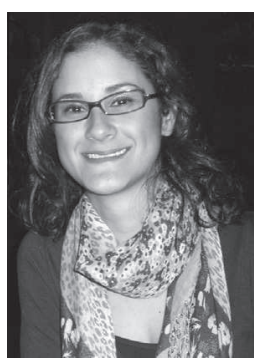

Mary R. Schurgot is a Cybersecurity Research Engineer in the Internet and Cybersecurity Research Department at LGS Bell Labs Innovations. She received her Ph.D. from Stevens Institute of Technology in 2012. In 2006 she completed her B.E. degree with high honors and in 2008 her M.E.E. with a concentration in wireless communications, both degrees from Stevens. She previously contributed to research projects while with BAE Systems, Inria, and MIT Lincoln Laboratory. Her current research interests are focused in the areas of wireless network modeling and optimization as well as military communications. She is a member of IEEE and SWE.

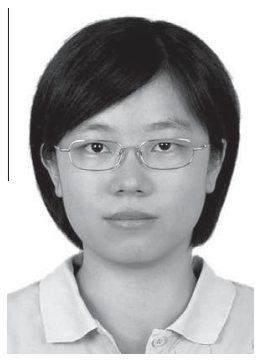

Qi Wang is a Ph.D. candidate in the Institute for Computing Technology at the Chinese Academy of Sciences in Beijing, China. In 2010, she received a 1-year fellowship from INRIA under the joint program with the Chinese Academy of Sciences to pursue her research within the SWING team of INRIA, at CITI laboratory, INSA Lyon, France. She is currently visiting IRIT laboratory at University of Toulouse because she is a recipient of the 2012 EIFFEL doctoral fellowship from the French Ministry of Foreign Affairs. Her research focuses on performance evaluation of wireless networks for delay sensitive applications. 


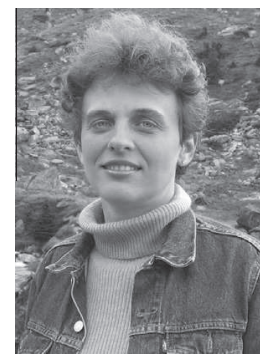

Cristina Comaniciu received the M.S. degree in electronics from the Polytechnic University of Bucharest in 1993, and the Ph.D. degree in electrical and computer engineering from Rutgers University in 2001. From 1998 to 2001 she was with the Wireless Information Network Laboratory (WINLAB) at Rutgers University working towards her Ph.D. thesis. From 2002 to 2003 she was affiliated with the Electrical Engineering Department at Princeton University as a postdoctoral fellow. Since 2003 she is with Stevens Institute of Technology, where she is currently associate professor in the Electrical and Computer Engineering Department. She is a recipient of the WINSection 2004 award. She won the 2007 IEEE Marconi Best Paper Prize Award in Wireless Communications for a paper co-authored with $\mathrm{H}$. Vincent Poor.

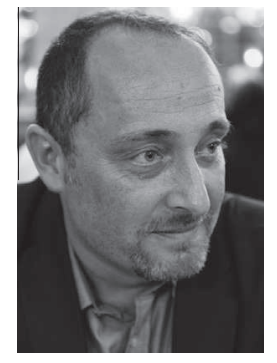

Jean-Marie Gorce is a professor of Wireless Communications at the National Institute of Applied Sciences (INSA), Lyon, France. He joined INSA as an associate professor in 1999, becoming full Professor in 2008. Since September 2009, he is head of CITI laboratory, a leading research group working on telecommunications in Lyon, France. Prof. Gorce received the Dipl. Ing. (M.Sc.) degree in electrical engineering (1993) and the $\mathrm{PhD}$ degree (1998) from INSA Lyon. He is a member of INRIA and the ADR Selfnet, a joint lab between INRIA and Alcatel Lucent. His main research concerns wireless communications focusing on realistic modeling and performance evaluation of infrastructure-based and ad hoc networks. 